\title{
Revisão das espécies de Melipona do grupo fuliginosa (Hymenoptera, Apoidea, Apidae, Meliponini)
}

\author{
João M. F. Camargo $0^{1,2} \&$ Silvia R. M. Pedro ${ }^{1}$
}

\begin{abstract}
${ }^{1,2}$ Departamento de Biologia, Faculdade de Filosofia, Ciências e Letras de Ribeirão Preto, Universidade de São Paulo, Av. Bandeirantes, 3900 , 14040-901 Ribeirão Preto-SP, Brasil.jmfdcama@usp.br; silviarmp@ffclrp.usp.br

${ }^{2}$ Pesquisador do $\mathrm{CNPq}$
\end{abstract}

\begin{abstract}
Revision of the species of Melipona of the fuliginosa group (Hymenoptera, Apoidea, Apidae, Meliponini). Three species are recognized: Melipona (Michmelia) fuliginosa Lepeletier, 1836, widely distributed, from Suriname and French Guiana to southeastern Brazil and northwestern Argentina, M. (Michmelia) titania Gribodo, 1893 (revalidated), endemic to western Amazon and M. (Michmelia) fallax sp. nov., from northwestern Ecuador to Central America. Melipona fuliginosa differs from the other species by the pilosity of metasomal terga II-V, dense and plumose, in both male and workers, and the first tarsomere of leg III of the male wider than long. In M. titania and M. fallax sp. nov., the pilosity of terga II-V is scarce and simple, non-plumose, and the first tarsomere of leg III of the male is as long as wide or longer. Workers of Melipona titania and M. fallax sp. nov. differ by the shape of the penicillum, which is strongly sinuous in $M$. titania, and in the males of $M$. fallax sp. nov. the internal orbits of the eyes are parallel, while in M. titania the orbits are convergent below. New geographic records, bionomic data and an identification key for the species are presented. In addition, comments are given on geographic patterns and tergal glands of the queens.
\end{abstract}

KEYWORDS. biogeography; nest; stingless bees; taxonomy; tergal glands.

\begin{abstract}
RESUMO. Revisão das espécies de Melipona do grupo fuliginosa (Hymenoptera, Apoidea, Apidae, Meliponini). Três espécies são reconhecidas: Melipona (Michmelia) fuliginosa Lepeletier, 1836, de larga distribuição, do Suriname e Guiana Francesa até o sudeste do Brasil e noroeste da Argentina, M. (Michmelia) titania Gribodo, 1893 (revalidado), endêmica do oeste da Amazônia, e M. (Michmelia) fallax sp. nov., do noroeste do Equador até a América Central. Melipona fuliginosa distingue-se pela pilosidade dos tergos metassomáticos II-V, tanto do macho como da operária, densa e plumosa, e pelo primeiro tarsômero da perna III do macho mais largo que longo. Em M. titania e M. fallax sp. nov., a pilosidade dos tergos II-V é escassa e simples, não-plumosa, e o primeiro tarsômero tão longo quanto largo ou mais longo que largo. Operárias de Melipona titania e M. fallax sp. nov. separam-se pela forma do penicilo, que é fortemente sinuoso em $M$. titania, e nos machos de $M$. fallax sp. nov. as órbitas internas dos olhos são paralelas, enquanto em $M$. titania as órbitas são convergentes embaixo. Novos registros geográficos, dados bionômicos e uma chave para identificação das espécies são apresentados. Adicionalmente, são feitos comentários sobre o padrão biogeográfico e sobre as glândulas tergais das rainhas.
\end{abstract}

PALAVRAS-CHAVE. Abelhas-sem-ferrão; biogeografia; glândulas tergais; ninho; taxonomia.

Melipona (Michmelia) fuliginosa Lepeletier, 1836, é uma espécie de tamanho avantajado (ca. 12-14 mm), a maior dentre as espécies de Melipona, e de cor negra; foi descrita de Caiena, Guiana Francesa. Vários nomes têm sido considerados como sinônimos juniores de M. fuliginosa: M. flavipennis Smith, 1854, M. titania Gribodo, 1893, M. opposita Gribodo, 1893, e M. bomboides Dominique, 1898 (Schulz 1904; Moure 1960; Schwarz 1932; Moure 1975; Rasmussen et al. 2007).

Aparentemente desconhecendo a Melipona fuliginosa de Lepeletier, de Caiena, Smith (1854) descreveu M. flavipennis com base em um macho, do Pará, Brasil. Mais tarde, Gribodo (1893), baseando-se na descrição de Smith, e também sem conhecer a descrição de M. fuliginosa, encontrou diferenças notáveis na pilosidade do abdômen, suficientes para descrever duas novas espécies, $M$. opposita, baseando-se em um macho, de Caiena, e M. titania, em uma operária de La Rioja, Argentina. Com base em uma operária, também de Caiena, Dominique (1898) descreveu M. bomboides. Mais tarde, Schulz (1904), ao comparar espécimens do Suriname, identificados por ele como
M. flavipennis, com exemplares etiquetados como M. titania no Museu de Munique, supôs que se tratassem da mesma espécie. Esta sinonímia foi aceita também por outros autores (Ducke 1910, 1916; Cockerell 1919; Schwarz 1932) e ratificada por Moure (1960; notas in litt.), que reestudou o material-tipo de Gribodo, inclusive de M. opposita, também incluída como sinônimo júnior de M. flavipennis. Foi somente em 1975 (p. 621, 622), ao revisar o material de Lepeletier, que Moure verificou a correspondência entre $M$. fuliginosa e $M$. flavipennis; destacou, entretanto, que o exemplar considerado por ele como o holótipo de M. fuliginosa, é uma operária, e não um macho.

Schwarz (1932: 273) comentou que, dentro de uma área de distribuição tão extensa, seria de se esperar algumas diferenças, mas embora tenha encontrado variações na forma da tíbia e basitarso da perna posterior e pontuação do tergo II ao comparar um macho do Peru com o tipo de M. flavipennis Smith no British Museum, ele não optou por considerá-las como espécies diferentes. O exame de espécimens, depositados na RPSP e outras coleções, provenientes de diversas 
localidades, também revelou descontinuidades relevantes, indicando que, ao menos 3 espécies distintas vinham sendo consideradas como M. fuliginosa, conforme já antecipado, em parte, por Camargo \& Pedro (2007: 398), o que levou a esta pequena revisão.

\section{MATERIALE MÉTODOS}

A maior parte dos exemplares examinados está depositada na Coleção Camargo (RPSP), sediada no departamento de Biologia da Faculdade de Filosofia, Ciências e Letras de Ribeirão Preto, Universidade de São Paulo, Brasil. As seguintes instituições e pesquisadores também forneceram exemplares para este estudo: AMNH - American Museum of Natural History, Nova Yorque, EUA, Dr. Jerome G. Rozen; DZUP Coleção Moure, Departamento de Zoologia, Universidade Federal do Paraná, Curitiba, PR, Brasil, Dr. Gabriel A. R. Melo; STRI - DWR Collection at Smithsonian Tropical Research Institute, Panamá, Dr. David Roubik; MUSM - Departamento de Entomologia, Museo de Historia Natural, Universidad Nacional Mayor de San Marcos, Lima, Peru, Dr. G. Lamas, C. Rasmussen; RASM - Coleção Claus Rasmussen; MZUSP - Museu de Zoologia, Universidade de São Paulo, Rodrigo Gonçalves.

A terminologia e forma de apresentação (diagnose, dimensões, forma e proporções, etc.) seguem Camargo \& Pedro (2005). Nas descrições, o metaposnoto é referido como área basal do propódeo. Os tergos metassomáticos são indicados como TI, TII, etc. A distância interocelar foi medida entre a margem interna do ocelo lateral e o ocelo médio, e a distância ocelorbital refere-se à menor distância entre a margem externa do ocelo lateral e a margem do olho.

As mensurações foram feitas em estereomicroscópio Leica MZ8 e os desenhos com auxílio de câmara clara.

No item "Material examinado", foram transcritos os dados das etiquetas de procedência, apenas foram padronizadas as indicações de meses (algarismos romanos, caixa alta) e anos (quatro algarismos). Países e subdivisões (estado, departamento, províncias, etc.) foram listados de oeste para leste e de norte para sul, conforme Zanella et al. (2000).

\section{Chave para operárias e machos das espécies do grupo fuliginosa.}

As espécies do grupo fuliginosa podem ser facilmente reconhecidas dentre as demais espécies de Melipona pela combinação dos seguintes caracteres: porte corporal grande (comprimento do corpo ca. $12 \mathrm{~mm}$, largura máxima da cabeça entre 4,6 e 5,1 mm); integumento preto, sem manchas amarelas; pilosidade preta em todo o corpo; plumosa, ereta, longa e abundante na cabeça e tórax; integumento polido e brilhante na face - principalmente na metade inferior - clípeo e mesoscuto; propódeo liso, brilhante, apenas com pontos pilígeros e algumas áreas superficialmente tesseladas; metade inferior da face glabra, apenas com algumas microcerdas simples, finas e eretas, esparsamente distribuídas pelo clípeo; tergos metassomais com cerdas longas, simples ou plumosas; asas amareladas; vértice da cabeça achatado, em aclive.
1. Tergos metassomais II-IV com cerdas simples (Fig. 3). Nos machos, apenas algumas cerdas plumosas entremeadas, o integumento visível sob a pilosidade; o limite entre a faixa basal glabra e a distal pilosa no tergo II com pontos grandes e bem marcados (Figs. 11, 12); o $^{\circ}$ tarsômero da perna III do macho tão longo quanto largo ou um pouco mais longo que largo (Fig. 14, 15) ... 2

Tergos metassomais II-IV com cerdas plumosas abundantes, além das cerdas simples (Fig. 4). Nos machos, o integumento escondido sob a pilosidade plumosa densa; o limite entre a faixa basal glabra e a distal pilosa no tergo II, praticamente liso, apenas com pontos superficiais e espalhados (Fig. 10); o $1^{\circ}$ tarsômero da perna III do macho mais curto que largo (Fig. 13). Norte e sudeste da América do Sul até o noroeste da Argentina (Fig. 22) ...................................... M. fuliginosa Lepeletier

2. Nas operárias, o penicilo mais largo na base e as cerdas posteriores em curva simples (Fig. 8). Nos machos, as órbitas internas dos olhos paralelas (Fig. 6); basitarso III como na Fig. 16. Noroeste do Equador até a Nicarágua (Fig. 22) M. fallax sp. nov.

Nas operárias, o penicilo mais estreito na base e as cerdas posteriores fortemente sinuosas (Fig. 9). Nos machos, as órbitas internas dos olhos convergentes embaixo (Fig. 7); basitarso III como na Fig. 15. Oeste da Amazônia (Fig. 22) ... .. M. titania Gribodo

\section{Melipona (Michmelia) fuliginosa Lepeletier, 1836}

Melipona fuliginosa Lepeletier 1836: 423; Spinola 1840: 124; Smith 1854: 404; Dalla Torre 1896: 578; Moure 1975: 615, 621-622 [partim]; Mori \& Pipoly 1984: 340; Camargo 1988: 352, 354; 1994: 50; Camargo et al. 1988: 155 [partim]; Roubik 1989: 28 [partim: Amazonas]; 1990: 580; 1992: 518 [partim]; ?1995: 68; 1996: 180;?Roubik \& Moreno 1990: 428; Garcia et al. 1992: 256 [partim: Amazonas]; Rêgo 1992a: 382, 383; 1992b: 74, 76, 77; Oliveira et al. 1995: 16, 17, 18; ?Melo 1996: 208; Nogueira-Neto 1997: 46 [partim, Amazonas]; Brown \& Albrecht 2001: 626, 627, 628, 629, 630; Oliveira 2001: 210, 216; 2002: 615, 629; Serrão 2001: 380; Fernandes-Salomão et al. 2002: 230; ?Kerr 2002: 6, 8; Velthuis et al. 2003: 172, 173, 174, 175; Cortopassi-Laurino 2004: 1-3; 2005: 37.

?Melipona fuscata, Spinola 1840: 130; non Lepeletier 1836.

?"urussú boi", "urussú preto", Castello Branco 1845: 49, 56-57; Papavero 1999: 3, 5. Papavero \& Teixeira 2000: 3, 4.

Melipona flavipennis Smith 1854: 406-407; Gribodo 1893: 251, 252 , 253; Dalla Torre 1896: 578; Schulz 1904: 816-817; Ducke 1910: 367; 1916: 16, tabela após página 28, 37, 170-171 [partim]; 1925 345, 357, 445-446 [partim]; Marianno 1911: 58-59; Cockerell 1919: 205 [partim: Mapiri, Bolivia]; Lutz \& Cockerell 1920: 493 [partim]; Schwarz 1932: 257, 265, 267, 268, 270-275, 298, 315, 345, 346, 424 [partim]; 1938: 439, 448 [partim]; ?1944: 415; 1948: 113, 120 [?partim]; Kerr 1948: 185, 251 [partim]; 1951: 288; Nogueira-Neto 1950: 307, 327, 328, 340, 341, 343, 351, 352, 355, 362, 363; 1970a: 417; 1970b: 34, 40, 41, 47, 48, 73, 285, 289, 334; Kerr \& Laidlaw 1956: 129; Moure et al. 1958: 488; Moure 1960: 15; Kerr et al. 1967: 137, 138, 139, 140, 141, 143; Mercado 1968: 4; Pisani et al. 1969: 98, 101, 102, 104, 105, 106; Cunha 1973: 27, figuras 2-7; 1991: 35, 43, 44, 45, 46, 47, 48, 49; Velthuis 1976: 41; Wille 1976: 136 [partim: Venezuela, Brasil, Bolivia]; Pisani et al. 1977: 234, 240, 241, 242, 243, 244; Engel \& Dingemans-Bakels 1980: 344, 347; Sakagami 1982: 364; 
Ramalho et al. 1991: 227, 231; Lenko \& Papavero 1996: 267, 273, 280; Velthuis 1997: 13; ?Ihering 2002: 329, 448, 558.

Melipona opposita Gribodo 1893: 253-254; Dalla Torre 1896: 582; Moure 1960: 15.

Melipona bomboides Dominique 1898: 58; Michener 1987: 6-7.

Melipona titania, Ducke 1901: 64; 1902a: 418; ?Ihering 1903: 279; ?1930: 702; non Gribodo 1893.

Melipona (Melipona) titania, Ducke 1902b: 288, 294-295; non Gribodo 1893.

Melipona fulvipennis [sic], ?Schwarz 1944: 416.

Melipona fluvipennis [sic], Kerr 1951: 298.

Melipona (Micheneria) flavipennis, Kerr \& Esch 1965: 536; Kerr 1969: 123, 124, 166

Melipona (Michmelia) fuliginosa, Moure 1992: 36 [partim]; Silveira et al. 2002: 89; Rasmussen et al. 2007: 60; Camargo \& Pedro 2007: 398 [partim].

Diagnose (operárias e machos). Pilosidade plumosa abundante nos tergos II-V, além das cerdas simples (Fig. 4); nos machos, a pilosidade muito densa nos tergos II-IV, recobrindo por completo o integumento subjacente (Fig. 10). Basitarso III do macho levemente encurvado e estreitado para a base, e o $1^{\circ}$ tarsômero curto e largo, seu comprimento ca. 0,8x sua largura máxima, com o canto póstero-distal fortemente projetado (Fig. 13). Bifurcação de $\mathrm{M}+\mathrm{Cu}$ coincidente ou ligeiramente anterior a cu-a. No macho, os olhos convergentes embaixo (Fig. 5); o limite entre a faixa basal glabra e a distal pilosa do tergo II, praticamente liso, apenas com pontos superficiais e espalhados (Fig. 10).

Macho (Figs. 5, 8, 10, 13, 17-21; Tab. I). Exceto pelos caracteres listados na chave, diagnose e nas medidas relacionadas na Tabela I, o macho de $M$. fuliginosa não difere dos de $M$. titania e $M$. fallax, inclusive a genitália e os esternos pré-genitais são praticamente idênticos (Figs. 17-21).

Rainha (Tab. I). Cor e textura do integumento como nas operárias, apenas mais para o avermelhado na parte ventral e nas pernas. Pilosidade preta-pardacenta, plumosa, na fronte e tórax; metade inferior da face lisa, brilhante, com cerdinhas eretas, esparsas no clípeo. A parte anterior do tergo I, largamente glabra, apenas com estreita faixa apical com cerdas eretas plumosas e lisas intercaladas; no tergo II as cerdas lisas e as plumosas intercaladas um pouco mais longas e abundantes, como nas operárias; as cerdas plumosas mais ralas nos tergos III e IV e ausentes nos seguintes, apenas com cerdas simples. Uma larga depressão glabra, com o integumento amarelado, a cada lado do tergo I, como na Fig. 23 (ver comentários em Melipona titania, item "Discussão"). Além disso, as genas são bojudas embaixo. As medidas estão relacionadas na Tab. I.

Material-tipo. Na descrição de Melipona fuliginosa, Lepeletier (1836: 424) refere-se a um macho de Caiena, Guiana Francesa, depositado no "Musée de M. Serville" (trazido por Doumerc). Parte do material de Lepeletier foi incorporada à "Coleção Spinola", agora depositada no Instituto de Zoologia da Universidade de Turim. Aí se encontra o exemplar que foi considerado por Moure (1975: 621-622) como o espécimen-tipo de $M$. fuliginosa; trata-se, porém, de uma operária, de "Cayenne", e não um macho, e com etiqueta constando o nome de "Buquet" [Jean Baptiste Lucien] (provavelmente o vendedor),


Figs. 1-2. Entrada de ninhos. 1. Melipona fuliginosa Lepeletier, 1836 (Tapera, Lago Caurés, Amazonas, Brasil, ninho 816c); 2. Melipona titania Gribodo, 1893 (Pacaysapa, próximo de Moyobamba San Martin, Peru; foto de C. Rasmussen). Escalas $=1,0 \mathrm{~cm}$.

e não a do coletor, Dr. Doumerc [Adolphe], como mencionado por Lepeletier (1836: 424). (De acordo com Papavero 1971: 130-131, Doumerc esteve em Caiena e região no período entre dezembro de 1823 a agosto de 1824.) Da mesma forma, Smith (1854: 406-407) descreveu Melipona flavipennis com base em um macho do Pará, Brazil, que foi reestudado por Schwarz (1932: 273). Moure (in litt.), estudou um espécimen depositado no Natural History Museum (British Museum - acc. BMNH 17b 1064), com etiqueta de "type" que, porém, trata-se de uma operária de Santarém, Pará, Brasil ( $c f$. Camargo \& Pedro 2007: 398). O holótipo de Melipona opposita Gribodo, um macho, de Caiena, Guiana Francesa, foi examinado por Moure em Abril de 1958 (1960: 15; notas in litt., ao qual se refere como tendo pilosidade densamente plumosa nos tergos abdominais), e encontra-se depositado no Museo Civico di Storia Naturale 'Giacomo Doria', Gênova, Itália. O materialtipo de Melipona bomboides Dominique foi estudado por Rasmussen \& Camargo (in Rasmussen et al. 2007), que designaram como lectótipo e paralectótipos três operárias do rio Maroni, Guiana Francesa, coletadas por E. Bar, depositados no Museu de História Natural de Nantes, França (MHNN).

Material examinado. SURINAME. Marowijine: Anapaike (Rio Lawa), X.1963, B. Malkin (3 ops., MZUSP); Christian Kondre, X.1963, R. Malkin col. (12 ops., MZUSP); GUIANA FRANCESA. Kourou, 25 
km SW, 15.V.1989, on Mimosa, D. Roubik leg., BC-2487 AOZ (1 op.,


(1 op., STRI). BRASIL. Roraima: Sta. Maria do Boiaçu, SA-20, 614' 'W, 0³0'S, 19-20.VIII.1980, Camargo, Mazucato leg. (1 op., RPSP); Amapá: Vila Amazonas, 21-III-1964, C. E. \& E. S. Ross (1 macho, DZUP); Amazonas: Arimã (Lago Jacaré), rio Purus, SB. 20-v-


151 ops., mais material fixado, incluindo mais 2 machos, ninho $375 \mathrm{c}$, RPSP); Foz do rio Paduari, 61 ${ }^{\circ} 13^{\prime}$ W $02^{\circ} 06$ 'S, 03-05-VIII. 1999 , Camargo, Pedro, Mazucato leg. (4 ops. 997915, 997916, 997937, 997937, RPSP); Manaus, Andrade, Rod. AM. 10, Km 20, 24.IX.1977, C. Gondim leg. ( 1 op., RPSP); Tapera, lago Caurés, rio Negro, $62^{\circ} 11^{\prime} \mathrm{W}$, 01ํ19'S, 27-28.VII.1999, Camargo, Pedro, Mazucato leg. (5 ops., 997505-997558, 997510, RPSP); ibidem, idem (02 machos, 60 ops., mais mat. fixado, incluindo mais machos, rainhas, etc., ninho $816 \mathrm{c}$, RPSP); Tremendaí, rio Negro, SA-20, 64 $46^{\prime} \mathrm{W}, 29^{\prime}$ [sic]S, 2.VIII.80, Camargo, Mazucato leg. (2 ops., RPSP); Pará: Belém, X.1948, Warwick Kerr, colony 79 [Melipona flavipennis F. Smith worker Det. H. F. Schwarz] (1 op., RPSP); Coraci, 15 km NW Canindé, rio Gurupi, IV.1963, B. Malkin col. (1 op., MZUSP); Óbidos, VII.1959, F. M. Oliveira (4 ops, MZUSP); Pará [provavelmente = Belém], 4.XI.1900, Ducke leg., etiquetas adicionais: "2", "Melipona flavipennis Sm P. Moure det. 39" 1 op., MZUSP); ibidem, idem, etiquetas adicionais "3", "18.202", "Melipona flavipennis Sm. det. Ducke 1911", "Melipona flavipennis Sm P. Moure det. 39" (1 op., MZUSP); ibidem, idem, "4", "Melipona flavipennis Sm. det. Ducke 1911", "Melipona flavipennis Sm P. Moure det. 39" (1 op., MZUSP); Acre: Xapuri, 26.IX.1989, Dain leg., no. 7 (6 ops., 900029-34, RPSP); Rondônia: Costa Marques, S12 ${ }^{\circ} 19.466^{\prime}$ W64⒕397', 18.XI.1996, Brown, Boina, Vieira leg. (1 op., RO 3250, RPSP); Guajará-Mirim, S10³3.751' W64²45.463', 10.IX.1996, Brown, Boina, Vieira leg. (7 ops., RO 555-561, RPSP); ibidem, idem, S1049.464' W6454.282', 09.X.1996 (2 ops., RO 1806, 1809, RPSP); ibidem, idem, S1045.519' W644⒈989', 10.X.1996 (14 ops., RO 1946-1953, 1981-1986, RPSP); Mirante da Serra, S10 59.881' W62 ${ }^{\circ} 50.764$ ', 24.IX.1996, Brown, Boina, Vieira leg. (9 ops., RO 2249-2257, RPSP); ibidem, idem, S11 ${ }^{\circ} 01.516^{\prime}$ W62 $48.750^{\prime}$, 07.XI.1996 (1 op., RO 2972, RPSP); Ouro Preto, S1041.970' W62 ${ }^{\circ} 19.332^{\prime}, 17$. XII.1996, Brown, Boina, Vieira leg. (2 ops., RO 4189, 4190, RPSP); ibidem, idem, S1042.970' W62 23.405', 29.I.1997 (1 op., RO 4955, RPSP); São Miguel do Guaporé, Linha 25, $11^{\circ} 43.563$ 'S - 62 ${ }^{\circ} 44.233^{\prime}$ W, 20.IV.1997, Brown, Boina, Vieira leg. (1 op., RO-7799, RPSP); Maranhão: Igarapé Gurupi-Uma [provavelmente Una], Aldeia Araçu, $50 \mathrm{~km}$ E de Canindé, V.1963, Malkin col. (1 op., MZUSP); Bahia: Água Preta, 7-XII-1943, Pedrito Silva (2 ops., DZUP); Mato Grosso: N. S. Livramento, 23-28.III.1965, S. Laroca leg. (2 ops., RPSP); Goiás: Jataí, Fazenda Aceiro, X.1962, Exp. Dep. Zool. (1 op., MZUSP); Espírito Santo: Linhares, Parque Sooretama, V.1953, P. A. Teles Col. (3 ops., DZUP); São Paulo: Rincão, sem data [certamente anterior a 1913], anônimo, etiquetas adicionais: "1", "18201", "Melipona titania Friese det.", "Melipona 18.201 flavipennis Sm. = titania (Grib.) Ducke. Rincão (Les. S.P.) Ducke ver. 13.", "Melipona flavipennis Sm. P. Moure det. 940" (1 op., MZUSP). Bolívia. Santa Cruz: Santa Cruz de la Sierra, XII.1952, W. Kempff (2 ops., DZUP). ARGENTINA. Salta: Vespucio (=General Enrique Vespucio, nome atual

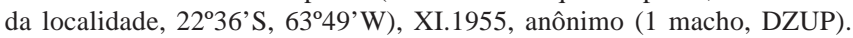

Distribuição geográfica e hábitat. Melipona fuliginosa, embora relativamente rara, é amplamente distribuída na América do Sul, desde as Guianas e Venezuela (Amazonas) até norte de Salta, Argentina. A oeste chega até o estado do Acre, e a leste, até a região de mata atlântica, na Bahia, Espírito Santo e São Paulo, Brasil (Fig. 22). Vive, preferencialmente, em matas altas e sombreadas. Castello Branco (1845) refere-se à ocorrência da uruçu-boi ou uruçu-preto no estado do Piauí, e pelas características, é possível que se tratasse da Melipona fuliginosa. O único registro existente para o estado de São Paulo é de um exemplar de "Rincão (Les. S.P.)", estudado por
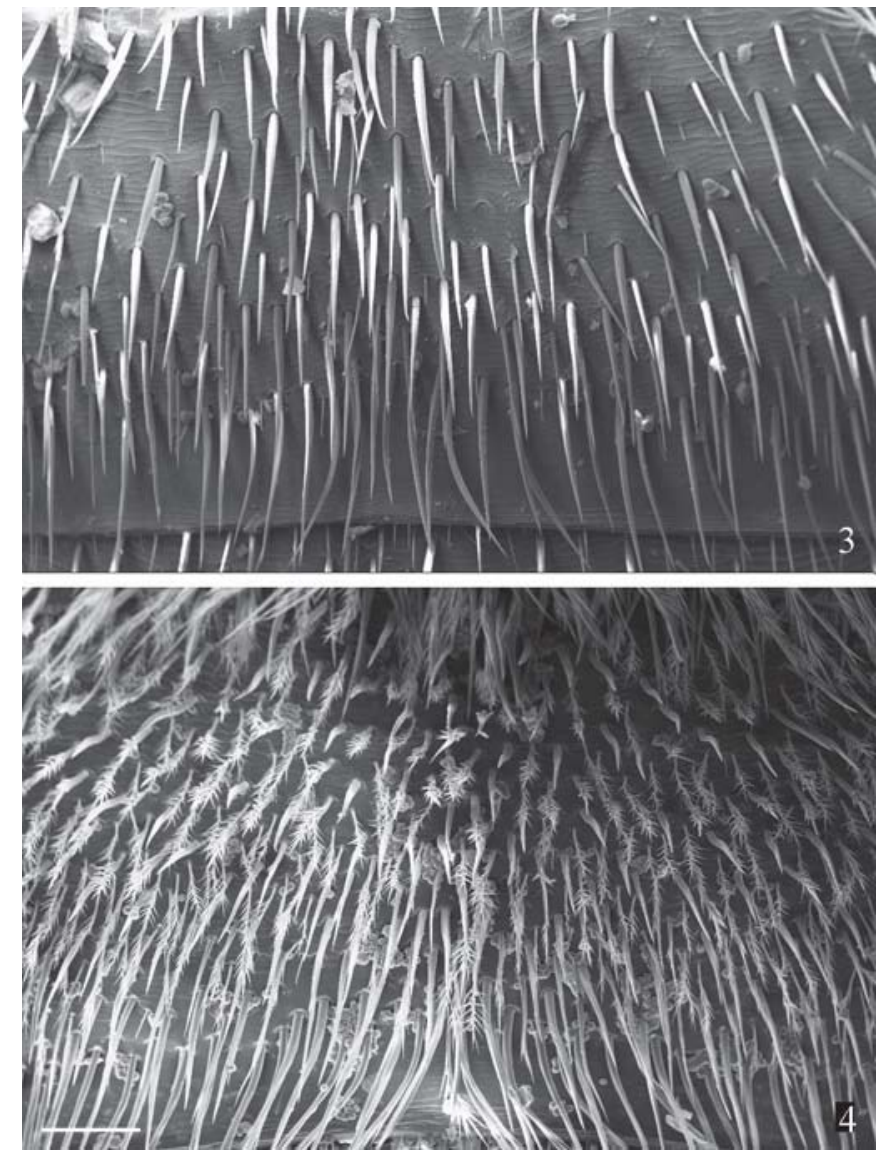

Figs. 3-4. Região discal do tergo III metassomal, operária. 3. Melipona fallax sp. nov. (Portobelo, Colon Prov., Panamá); 4. Melipona fuliginosa Lepeletier, 1836 (Tapera, Lago Caurés, Amazonas, Brasil, ninho 816c). Escala $=200 \mu \mathrm{m}$.

Ducke em 1913 (cf. Ducke 1916: 171) e que se encontra depositado no MZUSP. Kerr (2002: 8) menciona que sua presença foi registrada em São Paulo e Minas Gerais até o ano de 1940. Cortopassi-Laurino (2004) refere-se a uma informação de Paulo Nogueira-Neto de que existe um exemplar dessa espécie no MZUSP, coletado em 1950 no norte do estado de São Paulo (esse exemplar, todavia, não foi encontrado nessa coleção, R. Gonçalves, in litt., e segundo Paulo NogueiraNeto, comunicação pessoal, essa informação está equivocada). A ocorrência desta espécie na mata atlântica - Espírito Santo e Bahia, e sul de Goiás - norte de São Paulo, indica uma distribuição bem mais ampla desta espécie em tempos passados e atualmente "extinta" nas áreas mais devastadas. Faltam registros para o Brasil Central. Outro relato de extinção local refere-se à região leste da Bolívia (Beni - Santa Cruz), onde os índios Sirionó caçaram a "sucuasue" (provavelmente Melipona fuliginosa) até sua extinção (Stierlin e Szabo, informação pessoal a Roubik in litt. 2008).

Nidificação. Foram estudados, na natureza, apenas dois ninhos de M. fuliginosa, ambos em oco de árvores vivas. Um deles (no. de série 375c, de Arimã, rio Purus, AM, Brasil, 

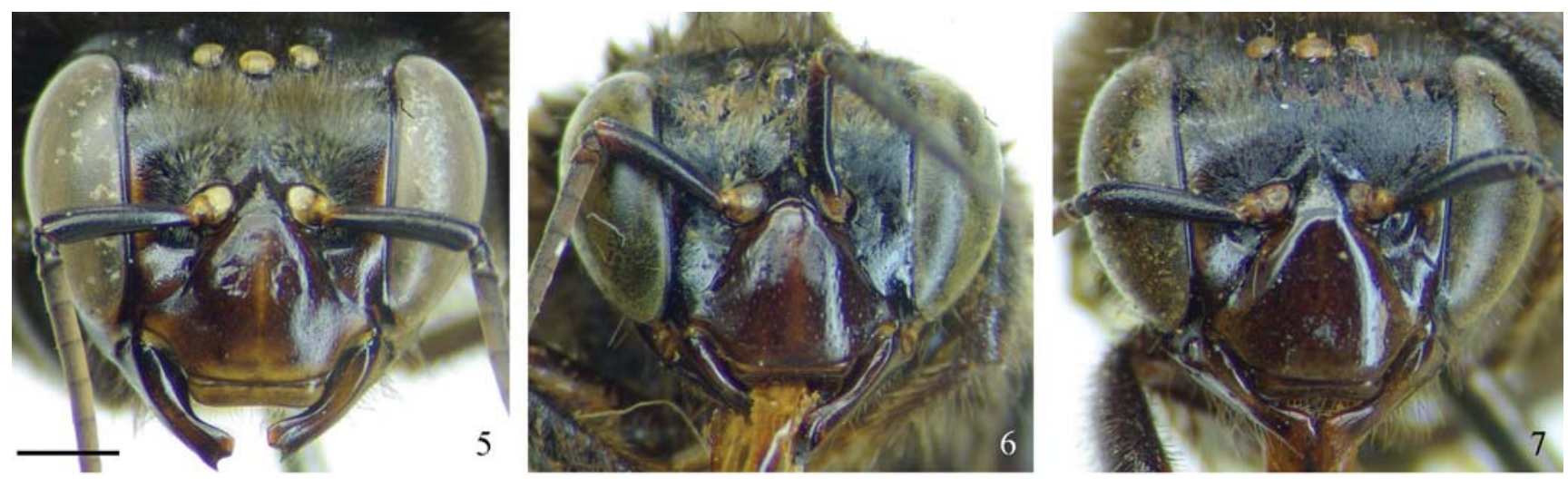

Figs. 5-7. Machos. 5. Melipona fuliginosa Lepeletier, 1836 (Tapera, Lago Caurés, Amazonas, Brasil, ninho 816c); 6. Melipona fallax sp. nov., parátipo (Chepo, Panamá Prov., Panamá); 7. Melipona titania Gribodo, 1893 (Napo river, Peru). Escala = 1,0 mm.

634ㄴ'W, 543'S, 8.II.1986, Camargo \& Mazucato) foi encontrado em mata de várzea, localizado em oco de "lombrigueiro" (provavelmente Crudia amazonica Benth, Leguminosae, Caesalpiniaceae) a 4,0 $\mathrm{m}$ acima do nível da água (período de rio cheio); o tronco media $60,0 \mathrm{~cm}$ de largura e o oco 16,0 cm de diâmetro. O outro (816c, de Tapera, lago Caurés, rio Negro, AM, Brasil, 62¹1'02"W, 01¹8'59"S, 27.VII.1999, Camargo, Pedro \& Mazucato), foi encontrado em mata de terrafirme (castanhal), em oco de tronco; a entrada situada a 80,0 $\mathrm{cm}$ acima do nível do solo; o tronco com $35,0 \mathrm{~cm}$ de largura e oco com $16,0 \mathrm{~cm}$ de diâmetro - o ninho todo ocupava $88,0 \mathrm{~cm}$ de extensão do oco.

Estrutura de entrada. Constituída de um tubo volumoso e irregular (no ninho 375c, com 10,0 cm de comprimento, $8,0 \mathrm{~cm}$ de diâmetro na base e $5,0 \mathrm{~cm}$ no ápice e no $816 \mathrm{c} \mathrm{com} 4,0 \mathrm{~cm}$ de comprimento, Fig. 1). O pavilhão de entrada, aproximadamente em forma de trompete, levemente afunilado até formar uma passagem com ca. $1,3 \mathrm{~cm}$ de diâmetro; a borda inferior mais alargada e projetada, formando uma pequena rampa de pouso e decolagem. Sem ornamentação em forma de estrias ou outros desenhos, construída exclusivamente com sementes, coladas umas as outras com a polpa que as revestem, avermelhada e pegajosa, de forma irregular, formando uma massa compacta muito dura (não solúvel em terebintina e quando queimada não produz chama e nem derrete, ao contrário da resina e cerume, respectivamente). No ápice do tubo, as sementes recentes tinham uma coloração avermelhada/lacre, em forte contraste com o restante do tubo, enegrecido pela oxidação. As sementes, pelo tamanho e formato - ovais achatadas, com 2,40 x 1,5 mm no ninho $375 \mathrm{c}$, e, 2,15 x 1,20 mm no ninho $816 \mathrm{c}-$ provavelmente sejam procedentes de Coussapoa spp (Cecropiaceae). Garcia et al. (1992) observaram várias espécies de Melipona coletando, intensamente, sementes de Coussapoa asperifolia magnifolia (Trécul) Akkermans \& Berg. Em um ninho estudado em Xapuri, Acre, CortopassiLaurino (2004) também observou intensa coleta de sementes avermelhadas e sua aplicação na entrada e em outras partes do ninho, mas as sementes não foram identificadas. $\mathrm{O}$ acesso ao interior do ninho se dá através de uma galeria com 1,8-2,0 $\mathrm{cm}$ de diâmetro interno e com grossas paredes $(0,5-0,8 \mathrm{~cm}$ de espessura) de resinas e sementes. No ninho 816c, cuja entrada situava-se na parte inferior do ninho, a galeria atravessava o batume e se prolongava até a parte inferior da região de crias, embaixo do invólucro.

Batume. No ninho 816c, o batume inferior, com ca. 3,0-4,0 $\mathrm{cm}$ de espessura, era constituído principalmente de resinas, sementes, alguma terra e exinas, não atravessado por canais regulares, mas apenas por pequenas galerias de 2,0-4,0 $\mathrm{mm}$ de diâmetro, muito irregulares e anastomosadas. Na parte superior não havia batume, embora o oco continuasse por mais de $1,0 \mathrm{~m}$.

Potes de armazenagem. De mel e pólen, misturados, localizados acima da região de crias; medindo ca. $3,5-5,0 \mathrm{~cm}$ de altura por 2,5-3,0 cm de diâmetro, no ninho $375 \mathrm{c}$, e 6,5-7,0 x 5,0 $\mathrm{cm}$ no ninho 816c; as paredes com 1,0-2,5 $\mathrm{mm}$ de espessura, construídas de cerume marrom-escuro e muito endurecidas. Em alguns potes mais velhos, as paredes chegavam a ser de resinas quase puras, formando finas camadas sobrepostas; nas paredes de alguns havia aplicações de sementes. Alguns potes isolados, mas a maioria construída com as paredes geminadas, formando massas de 4-5 ou mais potes. No ninho 816 c, havia ca. 30 potes, vários vazios, e outros parcialmente preenchidos com pólen ou mel. No ninho estudado por Cortopassi-Laurino (2004), havia mais de 100 potes, poucos com mel e muitos com pólen, e no total não havia mais que meio litro de mel.

Células de cria. Dispostas em favos horizontais sobrepostos, bem regulares, com espaços de aproximadamente 7,0-8,0 mm e sustentados por grossos pilares de cerume. Os casulos com ca. 14,23 x 7,74 mm. No ninho $816 \mathrm{c}$ havia nove favos, o maior com ca. 15,0 cm de diâmetro. Invólucro com 3-4 camadas de lamelas cerosas envolvendo todo o conjunto de favos.

População. No total, no ninho 816c, havia ca. 1350 células, contendo desde ovos até pupas emergentes. A população adulta com ca. 800 indivíduos, entre eles a rainha fisogástrica, quatro virgens e vários machos. Cortopassi-Laurino (2004) estimou a população adulta do ninho estudado no Acre em 700-1000 indivíduos, e contou 395 pupas em um único favo.

Comportamento de defesa. Reagem em grande número, voam rápido e mordem com insistência, mas não perseguem 
além de 5-10 m. No tubo de entrada, geralmente, se encontram 4-6 abelhas-guardas.

Outras notas bionômicas. Em seu estudo sobre as abelhas do Piauí, Castello Branco (1845) apresenta vários detalhes sobre o ninho da "urussú boi" ou "urussú preto", provavelmente a Melipona fuliginosa, e comenta que essas abelhas, que gostam de morar junto à superfície da terra, não costumam fazer muito mel, e não são boas para criação devido ao seu comportamento agressivo. Experimentos com colônias mistas foram feitos por Nogueira-Neto (1950), a partir de um ninho trazido de Belém-PA, Brasil.

Esta espécie tem o hábito de pilhar ninhos de outras abelhas sociais (Cortopassi-Laurino 2004), tal como M. titania, conforme relato de Nates-Parra \& Cepeda (1983). Venturieri (in litt. 2007) informa que observou, no Pará, M. fuliginosa invadindo ninhos de M. flavolineata Friese, 1900, "que estavam localizados a $100 \mathrm{~m}$ de distância de seu ninho, roendo, inclusive, a entrada da vítima que era menor". Também, tentativas de criá-las em caixas de madeira não foram bem sucedidas (Cortopassi-Laurino 2004; Venturieri in litt. 2007).

Nomes populares (veja Camargo \& Pedro, 2007): uruçuboi, uruçu-preto (Piauí, Brasil), mel-de-anta, tapii-ei, tapiieira, tapieira (oeste de São Paulo, Brasil), uruçu, mandury-preto (Mato Grosso, Brasil), Turuçu (Brasil), nara-buná-bisuki (índios Kaxinauá, Acre, Brasil), erereú-negra (Bolivia), sucuasue (índios Sirionó, Bolívia), tapir bee (Suriname; Roubik in litt. 2008).

Discussão. A identidade de Melipona fuliginosa Lepeletier, foi aqui interpretada considerando-se a descrição original e o material estudado proveniente da região próxima à localidade do tipo; pode ser reconhecida pela presença de pilosidade plumosa abundante nos tergos II-V, além dos demais caracteres listados na diagnose.

\section{Melipona (Michmelia) titania Gribodo, 1893}

Melipona titania Gribodo 1893: 251, 252, 253; Dalla Torre 1896: 584.

Melipona flavipennis, Ducke 1916: 171 [partim]; 1925: 445 [partim]; 1945: 128 [partim]; Cockerell 1919: 205 [partim]; Lutz \& Cockerell 1920: 493 [partim]; Schwarz 1932: 257, 265, 267, 268, 270-275, 298, 315, 345, 346, 424, plates I E, II E, IV E, VII D,E,F, IX A,B,C,D, X I [partim, espécimens do Peru]; 1938: 448 [partim: Peru]; 1948: 113, 120 [?partim]; Benoist 1933: 52; Moure 1944: 70; Dodson 1967: 20, 22, 58; Wille 1976: 136 [partim: Colombia, Equador, Peru]; non Smith 1854.

Melipona fuliginosa, Moure 1975: 621, 622 [partim]; Villa \& Vergara 1982: 151, 157, 159; Nates-Parra 1983: 158 [partim ?]; 1995: 24, 26, 27, 30 [partim ?]; 2001: 238, 245 [partim ?]; Nates-Parra \& Cepeda 1983: 69, 70, 74; Coloma 1986: 11, 92, 135 [partim: Napo - Coca, Pastaza - Puyo]; Vergara et al. 1986: 183; ?NogueiraNeto 1997: 355 [partim: Colombia]; Nates-Parra \& González 2000: 21; Roubik 2000: 273 [partim: Peru, Colombia]; Smith-Pardo 2003: 338; Nates-Parra et al. 2006: 29; non Lepeletier 1836.

Melipona (Michmelia) fuliginosa, Camargo \& Pedro 2007: 398 [partim]; non Lepeletier, 1836.
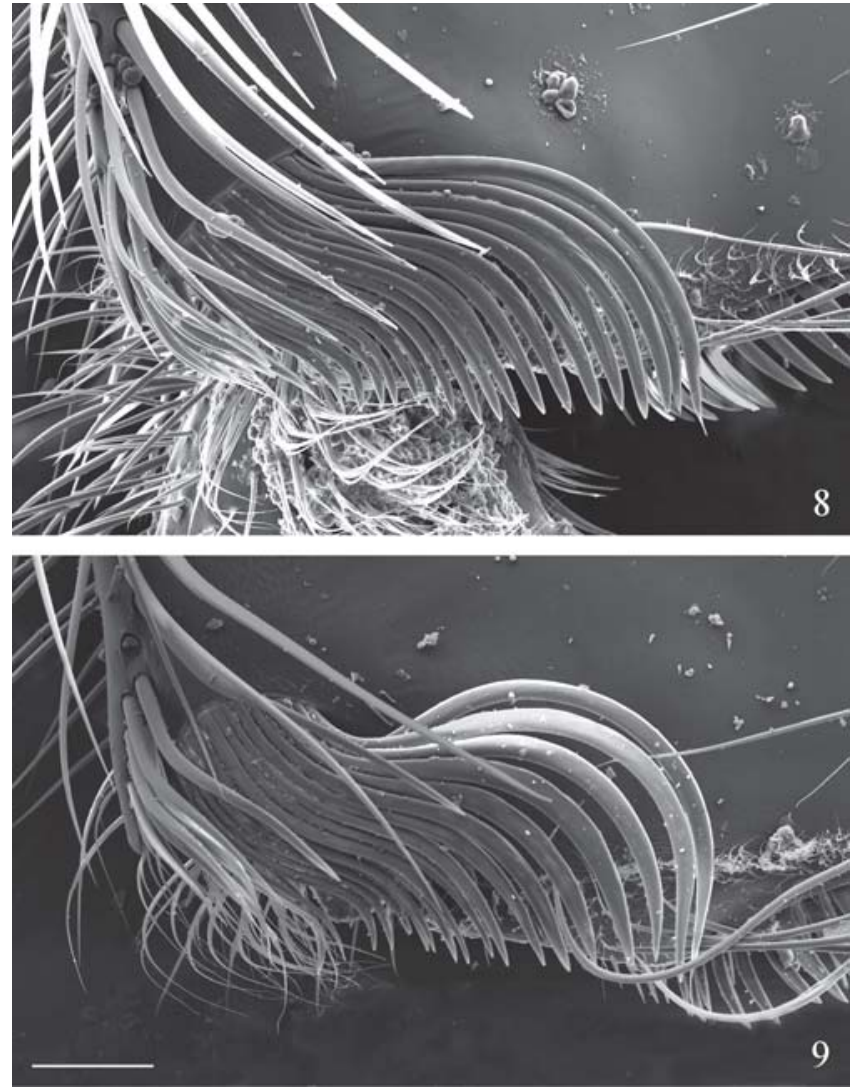

Figs. 8-9. Tíbia III, detalhe do penicilo, operária. 8. Melipona fallax sp. nov. (parátipo, Portobelo, Colon Prov., Panamá); 9. Melipona titania Gribodo, 1893 (Pacaysapa, near Moyobamba, San Martin, Peru). Escala $=0,02 \mathrm{~mm}$.

Diagnose (operárias e machos). Pilosidade nos tergos IIVI constituída apenas de cerdas simples (como na Fig. 3), esparsas, as plumosas estão presentes apenas em TI; nos machos, algumas poucas cerdas plumosas intercaladas às cerdas simples nos tergos I-IV, o integumento amplamente visível sob a pilosidade (Fig. 12). Nas operárias, o penicilo mais estreito na base e as cerdas posteriores fortemente sinuosas (Fig. 9). No macho, o basitarso III subretangular, de lados aproximadamente paralelos (Fig. 14); $1^{\circ}$ tarsômero da perna III mais longo que largo, seu comprimento ca. de 1,11,2x sua largura máxima (Fig. 14). Bifurcação de $\mathrm{M}+\mathrm{Cu}$ anterior a cu-a. Nos machos, as órbitas internas dos olhos convergentes embaixo (Fig. 7), o limite entre a faixa basal glabra e a distal pilosa no tergo II com pontos grandes e bem marcados (Fig. 12).

Macho (Figs. 7, 9, 12, 14; Tab. I). Apenas um macho foi visto, trata-se do mesmo espécimen estudado e figurado por Schwarz (1932, prancha IX, figs. A-D). A genitália foi dissecada por Schwarz e montada em bálsamo, entre lamínulas, de modo que as partes ficaram um pouco deformadas, mas de uma maneira geral, não difere daquela de $M$. fallax sp. nov. (Fig. 16). Nos demais caracteres, como em $M$. fallax sp. nov., exceto naqueles listados na chave e na diagnose. 
Rainha. A rainha de $M$. titania foi descrita e ilustrada por Schwarz (1932: 271-272, pranchas I-fig. E, VII-fig. D e X-fig. I), todavia ele não faz referência à pilosidade dos tergos. Pela fig. E, pode-se notar que a gena, embaixo, é pouco bojuda, semelhante a de $M$. fallax sp. nov.

Material-tipo. O holótipo, operária, de Melipona titania Gribodo, de La Rioja, Argentina, foi examinado por Moure (1960: 15 e notas in litt.) em Abril de 1958, e encontra-se depositado no Museo Civico di Storia Naturale 'Giacomo Doria', Gênova, Itália. É possível que a procedência desse exemplar esteja errada como já comentado por Ducke (1916: 171). Não se conhece nenhum outro registro dessa espécie na Argentina, há apenas registros para M. fuliginosa.

Material examinado. COLÔMBIA. Amazonas: Letícia, $65 \mathrm{~km} \mathrm{~W}$ Amacayacu Nat. Park, 17-30.IX.1986, D. Roubik leg. (1 op., 870357, RPSP); Meta: Villavo. [=Villavicencio], Jardim Botànico, Muestra 7, 640 m, 6.X.2004, A. Parra leg. (1 op., 18089, PARRA). EQUADOR. Pastaza: Puyo, VIII.1986, G. Onore leg. (2 ops., RPSP); Morona Santiago: Mendez, VII.1992, D. Roubik leg. (01 op., STRI); Zamora Chinchipe: Zamora, 3.VIII.1967, R. L. Dressler (1 op., DZUP). PERU. Loreto: Iquitos, XI.1964, G. H. Dodson, ex. Cattleya luteola, "65" (1 op., DZUP); Napo River, 20.VI.1920, H. Parish leg. [M. flavipennis Smith Det. Schwarz] (1 op., RPSP;1 macho, AMNH, com etiqueta adicional: "compared with type of flavipennis"); Peru [sem localidade especificada], sem data, anônimo, etiquetas adicionais: "5", "1.00L", "Melipona titania 1900 Friese det. Peru", "Melipona flavipennis Sm P. Moure det. 39" (1 op., MZUSP); San Martin: Moyobamba, XII.1964, C. H. Dodson, ex. flores de Bixa orellana (1 op., DZUP); Pacaysapa, nr. Moyobamba, 0616S/7647W, 24.I.2008, 860m, Rasmussen leg. (ops., ninho, RASM; 6 ops., RPSP); San Antonio de Cumbaza, 0625S/7624W, 500m, 28.I.2008, Rasmussen leg. (ops., ninho, RASM); Pasco: Sta. Rosa (R. Palcazu), 28.IX.1992, 500 mt., Pedro Hocking leg. (1 op., MUSM); Junín: Satipo, Capiri, 12.VIII.1976, R. Garcia leg. (1 op., MUSM); Cuzco: Pilcopata, 17.II.1993, L. Gonzales leg. (1 op., MUSM); Madre de Dios: Rio Los Amigos Park, 15-20.X.2003, D. W. Roubik leg. (1 op., STRI). BRASIL. Amazonas: Tefé, 1-4.XII.1961, F. M. Oliveira leg. (1 op., RPSP).

Distribuição geográfica e habitat. Acompanha as vertentes orientais da cordilheira andina, da Colômbia - Cundinamarca e Meta, até La Rioja, Argentina (?), sendo o registro mais a leste, de Tefé, Amazonas, Brasil (Fig. 22). Além das localidades dos espécimens listados em "Material examinado", também foram considerados, tentativamente, como pertencentes a esta espécie os registros para Archidona, Napo, Equador (Schulz, 1904), e Medina, Cundinamarca, Colômbia (Villa \& Vergara 1982) pela proximidade geográfica com as localidades dos espécimens estudados (ver, no final, "Comentários gerais sobre o padrão de distribuição geográfica").

Nidificação e outras notas bionômicas. Villa \& Vergara (1982) e Vergara et al. (1986) estudaram um ninho em Medina, Cundinamarca (Colômbia), mas não mencionam detalhes, apenas que estava em oco de árvore viva, e que não tinha tubo de entrada. Segundo Nates-Parra (1995) a entrada é constituída apenas de um orifício para passagem de uma só abelha.

Nates-Parra \& Cepeda (1983) mencionam que estas abelhas (M. titania ?, supostamente referindo-se ao ninho estudado em Medina) são muito mansas, não atacam humanos, porém são pilhadoras violentas, atacam até mesmo colméias de Apis,
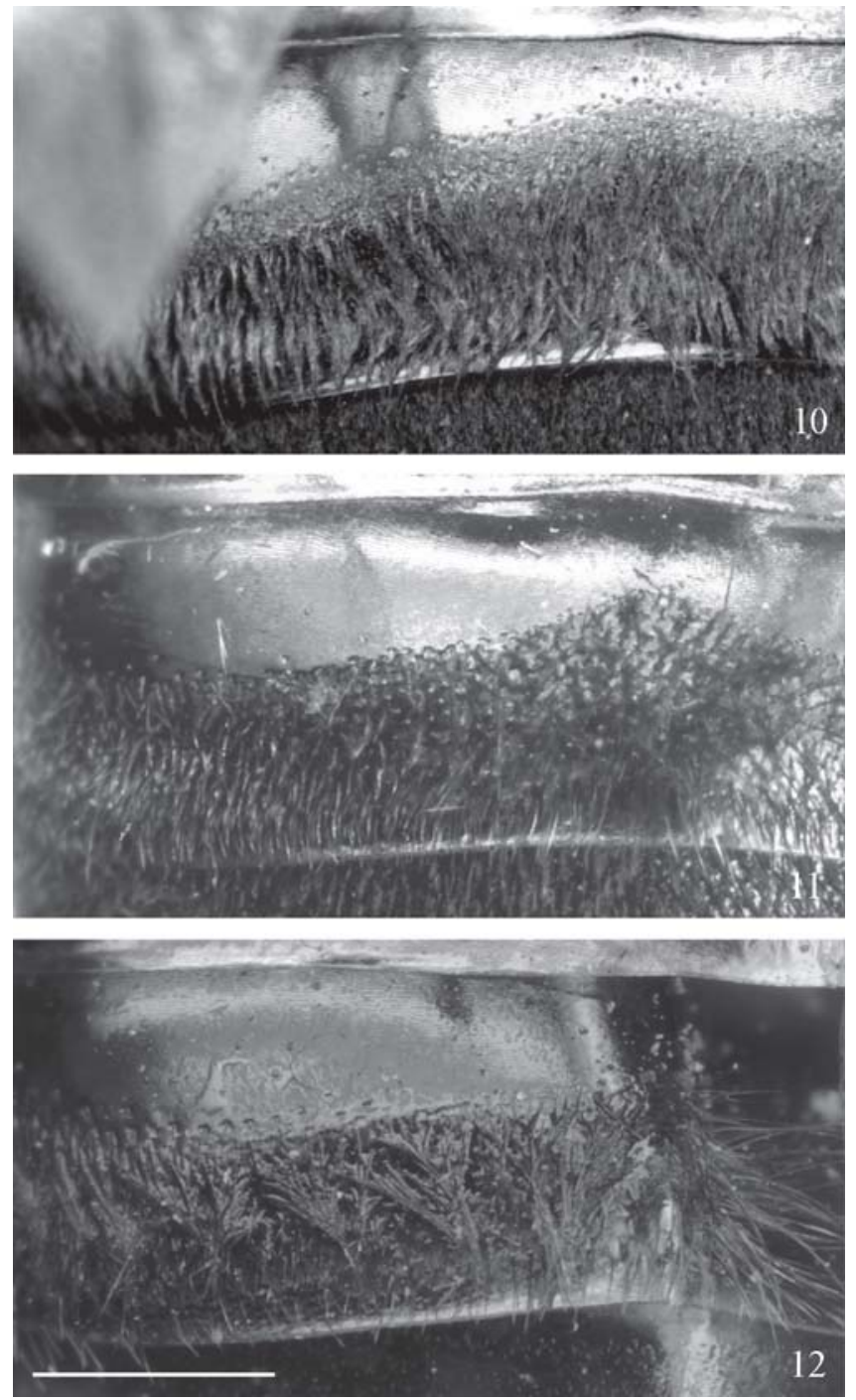

Figs. 10-12. Tergo II metassomal, macho. 10. Melipona fuliginosa Lepeletier, 1836 (Tapera, Lago Caurés, Amazonas, Brasil, ninho 816c); 11. Melipona fallax sp. nov., parátipo (Chepo, Panamá Prov., Panamá); 12. Melipona titania Gribodo, 1893 (Napo river, Peru). Escala $=1,0 \mathrm{~mm}$.

decapitando as operárias que saem em defesa do ninho. Rasmussen (in litt. 2008) informa que obteve dois ninhos de M. titania, ambos localizados em oco de árvore viva, um de San Antonio de Cumbaza, e outro de Pacaysapa, Moyobamba, San Martin, Peru; o primeiro morreu ao ser transferido para uma caixa, e o segundo foi conservado no próprio tronco; relata, ainda, que são agressivas, mordem, se enredam nos pêlos e vibram as asas e que a entrada dos ninhos forma um tubo constituído de sementes vermelhas e amareladas (Fig. 2) [provavelmente de Coussapoa sp., exatamente iguais às sementes encontradas no ninho $375 \mathrm{c}$ de M. fuliginosa, Purus, $\mathrm{AM}$. Do ninho obtido em San Antonio de Cumbaza, Rasmussen obteve mais de 2 litros de mel, além de uma quantidade grande que foi perdida durante a transferência do ninho, e que esta foi a maior quantidade de mel que ele obteve de um ninho de Meliponini no Peru. Dodson (1967) menciona 
Melipona titania (citada como M. flavipennis) como polinizador de Cattleya luteola no Peru, e Roubik (2000, citada como M. fuliginosa) como polinizador de Maxillaria, em Letícia, Colombia.

Nomes populares (veja Camargo \& Pedro, 2007): currunchos-grandes (Colômbia).

Discussão. A identidade de Melipona titania foi definida com base na descrição de Gribodo (1893) e, principalmente, nas informações de Moure (in litt.) sobre o tipo, que se refere, claramente, às "cerdas curtas, não plumosas", nos tergos abdominais. O material estudado por Schwarz (1932), proveniente de um ninho do Peru - Napo River, refere-se a Melipona titania, conforme revelado pelo exame do macho depositado no AMNH (este foi o espécimen utilizado por Schwarz 1932: 273, para comparação com o tipo de $M$. flavipennis Smith, e para os desenhos da genitália - prancha IX, figs. A,B,C,D). Melipona titania e M. fallax sp. nov. compartilham o mesmo padrão de pilosidade nos tergos, porém, as operárias destas espécies apresentam diferenças na forma do penicilo; nos machos, entretanto, as diferenças são mais sutis, conforme caracteres listados na chave e na diagnose.

Schwarz (1932: 274, prancha X, fig. I), ao estudar rainhas de $M$. flavipennis (=M. titania), chama a atenção para uma peculiaridade estrutural: uma forte depressão a cada lado do $1^{\circ}$ tergo metassomático; refere-se, também, a uma estrutura semelhante em rainha de $M$. (Michmelia) rufiventris Lepeletier, 1836. Na verdade, essas depressões contêm aberturas de glândulas; finas linhas concêntricas do integumento confluem para um ponto central onde desembocam canalículos excretores. Dissecações em rainhas de Melipona (Michmelia) sp. grupo rufiventris mostraram que os canalículos são ligados a grandes células, que formam um conjunto, que lembra um cacho de uvas (provavelmente correspondem às glândulas tergais unicelulares mencionadas por Guerino \& Oliveira 2002), logo abaixo da superfície da depressão do tergo. Essas depressões no tergo estão presentes não só em rainhas de $M$. titania, M. fuliginosa e M. fallax sp. nov., mas, possivelmente, em rainhas de todas as demais espécies de Michmelia e ausente nos demais subgêneros, constituindo-se, provavelmente, em uma forte apomorfia para esse subgênero. Todavia, segundo Roubik (in litt. 2008), rainhas de $M$. (Michmelia) panamica Cockerell, 1912, não possuem depressões no tergo I, comparáveis às de M. fallax sp. nov. Foi constatada a ocorrência da área glandular rebaixada nas seguintes espécies do subgênero Michmelia: Melipona fuliginosa, M. titania, M. fallax sp. nov., M. crinita Moure \& Kerr, 1950, M. paraensis Ducke, 1916, M. fulva Lepeletier, 1836, M. nebulosa Camargo, 1988, M. flavolineata Friese, 1900, M. rufiventris Lepeletier, 1836, $M$. sp. grupo rufiventris, $M$. capixaba Moure \& Camargo, 1994, M. fuscopilosa Moure \& Kerr, 1950, M. sp. grupo eburnea fuscopilosa, M. lateralis Erichson, 1848, M. seminigra abunensis Cockerell, 1912, $M$. seminigra merrillae Cockerell, 1919, M. scutellaris Latreille, 1811. Área glandular rebaixada está ausente nos subgêneros



Figs. 13-15. Basitarso e tarsômeros da perna III, macho. 13. Melipona fuliginosa Lepeletier, 1836 (Tapera, Lago Caurés, Amazonas, Brasil, ninho 816c); 14. Melipona titania Gribodo, 1893 (Napo river, Peru). 15. Melipona fallax sp. nov., parátipo (Chepo, Panamá Prov., Panamá) Escala $=1,0 \mathrm{~mm}$

Eomelipona (Melipona asilvai Moure, 1971, M. marginata Lepeletier, 1836, M. puncticollis Friese, 1902, M. schwarzi Moure, 1963), Melikerria (M. interrupta Latreille, 1811, M. fasciculata Smith, 1854, M. quinquefasciata Lepeletier, 1836) e Melipona (M. quadrifasciata Lepeletier, 1836, M. orbignyi Guérin, 1844).

\section{Melipona fallax sp. nov.}

Melipona flavipennis, Cockerell 1919: 205 [partim]; Lutz \& Cockerell 1920: 493 [partim]; Schwarz 1932: 273, 275 [partim: Zona do Canal e Costa Rica]; 1934: 6, 7; 1938: 448 [partim]; Kerr 1948: 185 [partim]; Michener 1954: 164; Wille 1961a: 7; 1961b: 118, $120,123,124,125,126,127 ; 1976: 126,127,128,136,138$ 144, 145, 146, 147 [partim, Costa Rica, Panamá]; Darchen 1969: 461, 462; Wille \& Michener 1973: 16, 28, 37, 51, 62, 257, 258259, 260-261; Wille \& Orozco 1975: 260; Roubik 1979: 414; Roubik 1981: 263-268 [partim: Panamá, Costa Rica]; Roubik \& Wheeler 1982: 127; non Smith 1854.

Melipona nigra (=flavipennis), Wille 1979: 253; non Lepeletier 1836. Melipona fuliginosa, Nates-Parra 1983: 158 [partim ?]; 1995: 24, 26, 27, 30 [partim ?]; 2001: 238, 245 [partim ?]; Roubik 1983: 331, $336,340,344,346,348,349,350,352 ; 1989: 28,29,68,69,86$, 103, 132, 137, 177, 181, 202, 208, 211, 416-417 [partim: Panamá, Costa Rica]; 1992: 498, 505, 508, 518, 523 [partim]; 1993: 548; 2000: 273, 275 [partim: Costa Rica, Panamá]; Wille 1983: 51, 54; Roubik \& Buchmann 1984: 2-10; Coloma 1986: 11, 36-40, 92, 113, 131, 135, 137, 138 [partim: Esmeraldas - La Sexta, Imbabura - Rocafuerte]; Roubik et al. 1986: 99, 101, 102, 103, 105; Camargo et al. 1988: 155 [partim]; Ramalho et al. 1990: 473; Garcia et al. 1992: 256 [partim: Panamá]; Sakagami et al. 

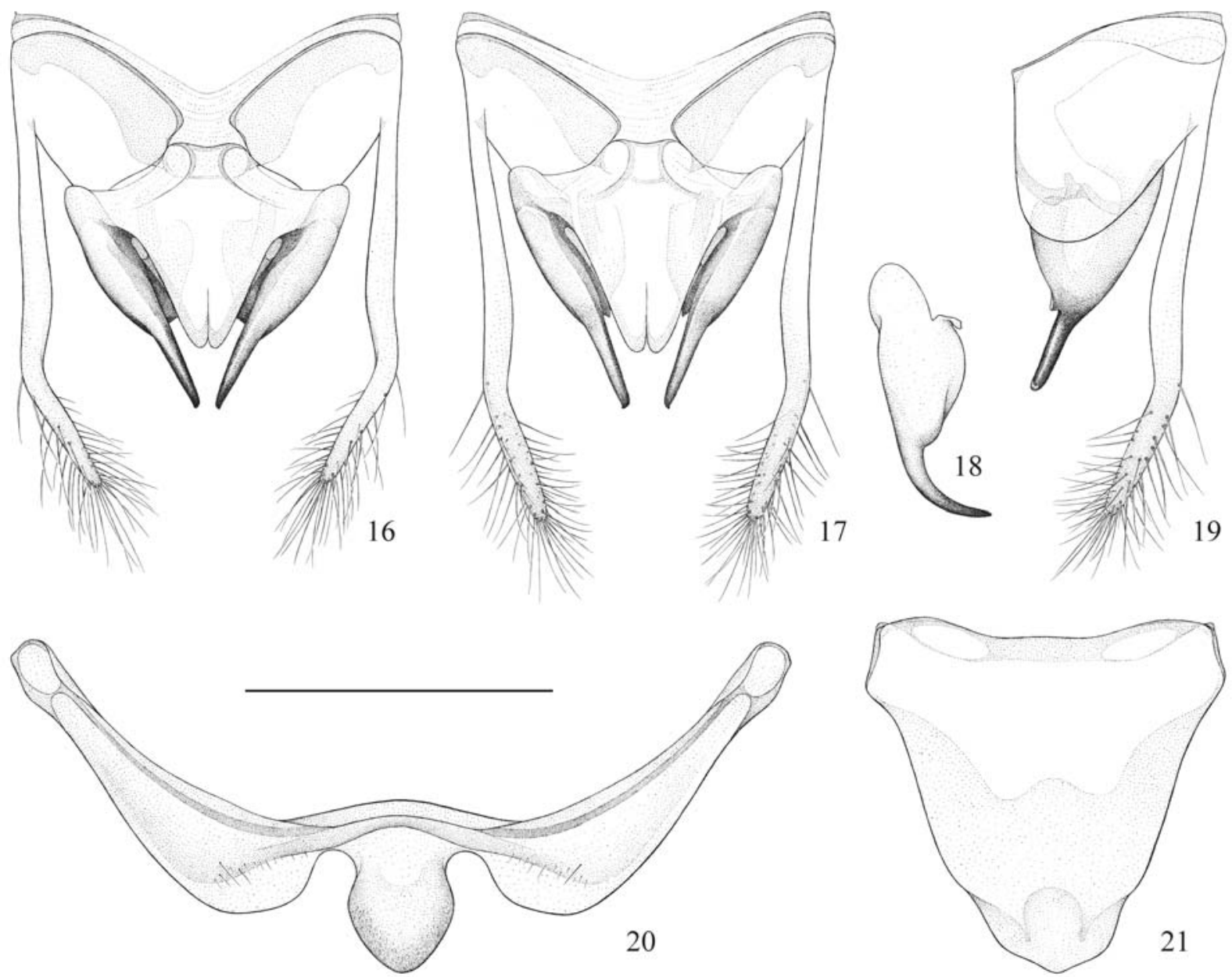

Figs. 16-21. Genitália e esternos pré-genitais, macho. 16. Melipona fallax sp. nov., parátipo (Chepo, Panamá Prov., Panamá), genitália, vista dorsal; 17-21. Melipona fuliginosa Lepeletier, 1836 (Tapera, Lago Caurés, Amazonas, Brasil, ninho 816c): 17. geinitália, vista dorsal; 18. detalhe do gancho, visto de perfil; 19. genitália, vista ventral; 20. esterno metassomal VI; 21. esterno metassomal VII. Escala 1,0 mm.

1993: 243; Griswold et al. 1995: 684, 689; Roubik et al. 1995: 204, 205, 206, 208; Biesmeijer 1997: 26, 27, 28, Bruijn \& Sommeijer 1997: 44; Nogueira-Neto 1997: 46 [partim, Panamá]; Crane 1999: 82, 289; Heard 1999: 187, 192; Gonzalez-B \& OspinaTorres 2000: 357; Pereboom \& Biesmeijer 2003: 43, 44, 45, 47; Pierrot \& Schlindwein 2003: 569; Barcelar-Lima et al. 2006: 344; non Lepeletier 1836;

Melipona cf. flavipennis, ?Parra 1984: 286.

Melipona (Michmelia) fuliginosa, Moure 1992: 36 [partim]; Camargo \& Pedro 2007: 398 [partim]; non Lepeletier 1836.

Diagnose (operárias e machos). Pilosidade nos tergos IIVI constituída apenas de cerdas simples (Fig. 3), as plumosas estão presentes apenas em TI; nos machos, algumas poucas cerdas plumosas intercaladas às cerdas simples nos tergos II e III, o integumento amplamente visível sob a pilosidade (Fig. 11). Nas operárias, o penicilo mais largo na base e as cerdas posteriores em curva simples (Fig. 8). No macho, o basitarso III subretangular, levemente estreitado para a base (Fig. 15); $1^{\circ}$ tarsômero da perna III aproximadamente tão longo quanto largo (Fig. 15). Bifurcação de $\mathrm{M}+\mathrm{Cu}$ anterior a cu-a. Nos machos, os olhos subparalelos (Fig. 6); o limite entre a faixa basal glabra e a distal pilosa do tergo II com pontos grandes e bem marcados (Fig. 11).

Holótipo, operária (Fig. 3, Tab. I).

Dimensões. Comprimento total aproximado, $11,70 \mathrm{~mm}$; da asa anterior, desde o ápice do esclerito Costal, 9,20 mm (incluindo a tégula, 10,40 mm); largura máxima da cabeça, 4,85 $\mathrm{mm}$; do TII 5,29 mm.

Cor do integumento. Preto, apenas o clípeo, pernas, disco do propódeo e tergos levemente mesclados de vermelho; flagelo ferrugíneo escuro, o $1^{\circ}$. flagelômero um pouco mais claro; margem dos esternos metassomais e do TI, castanhoferrugínea. Membrana das asas hialina, tingida de amarelo, mais fortemente nas células Radial e Marginal; microtríquias amarelas; veias amareladas.

Pilosidade. Quase toda preta, abundante e predominantemente plumosa; cerdas das peças bucais, amarelas; nos tarsômeros de todas as pernas, ferrugíneo- 


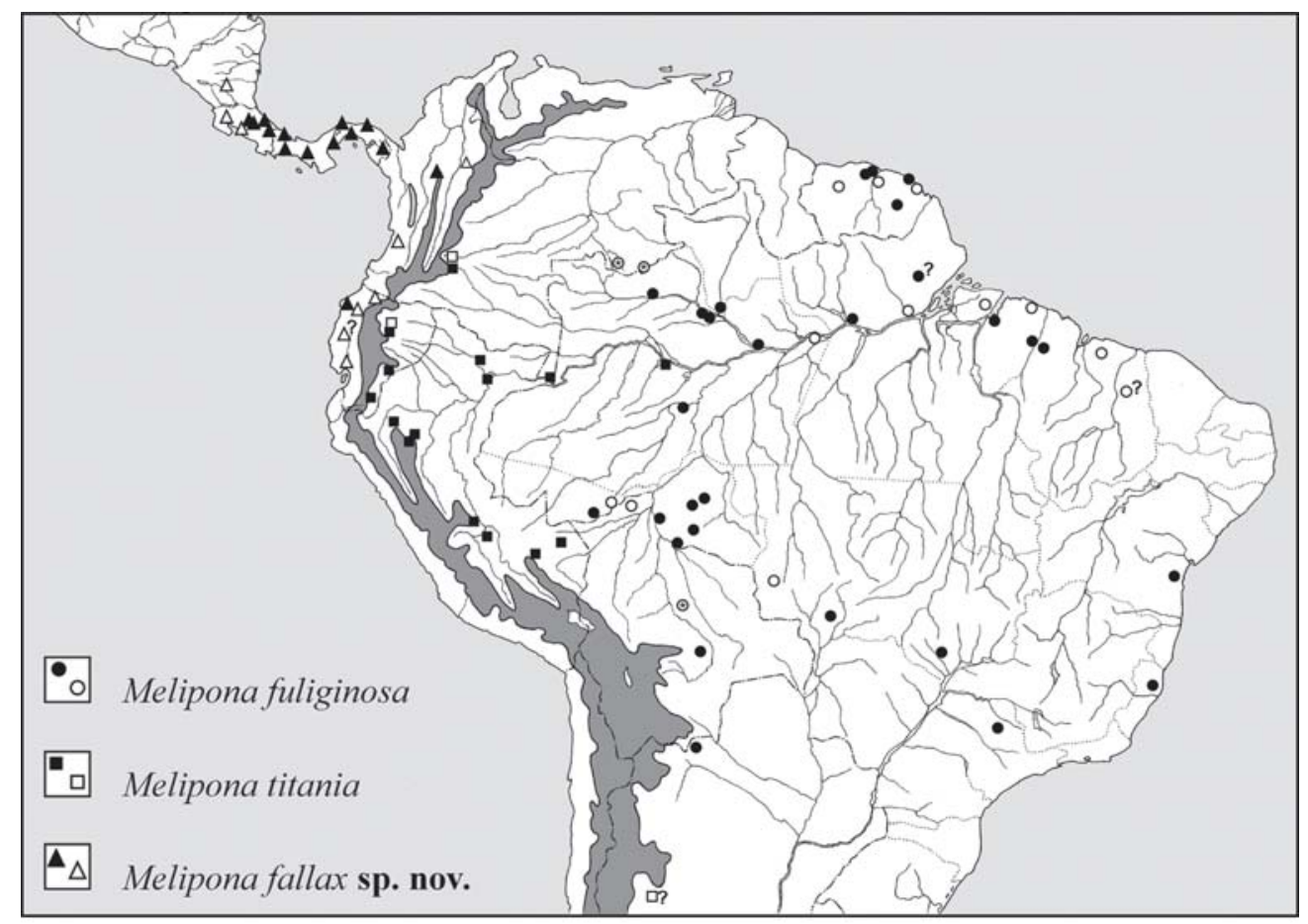

Fig. 22. Distribuição geográfica das espécies do grupo fuliginosa. Símbolos vazados representam dados de literatura; aqueles indicados com asterisco (*) foram obtidos no site Discover Life (http://www.discoverlife.org), e referem-se a exemplares depositados no AMNH, da Venezuela (Santa Lucia e Cerro de la Neblina, Puerto Chimo, Amazonas) e Bolivia (Rio Sajita, in the Chapare, Cochabamba).

enegrecidas; ápice das cerdas ventrais, pálido; tufos de cerdas plumosas mais claras, com a haste escura e os ramos de cor palha, nos lóbulos pronotais, parte anterior da tégula e lados do propódeo e pequenas áreas adjacentes. Pilosidade plumosa, longa e abundante na fronte e vértice, e encobrindo densamente o integumento do tórax; na fronte e vértice chegando até $0,88 \mathrm{~mm}$ de comprimento, nos lados dos mesepisternos com $0,83 \mathrm{~mm}$, no mesoscuto variando de 0,60 (disco) a 1,0 mm, e um pouco mais longa no ápice do escutelo $(1,1 \mathrm{~mm})$. Na metade inferior da face, apenas pequenas cerdas esparsas, simples, eretas, chegando a $0,22 \mathrm{~mm}$ de comprimento no disco do clípeo. Algumas cerdas plumosas decumbentes nas áreas paroculares aos lados e acima dos alvéolos antenais. No escapo, as cerdas simples e curtas, as mais longas não ultrapassando o diâmetro deste. As genas com cerdas simples, semi-eretas, com até um pouco mais de $1 \mathrm{~mm}$ de comprimento, e algumas cerdinhas plumosas, decumbentes, esparsas. $\mathrm{O}$ penicilo largo na base e as cerdas posteriores em curva simples (como na Fig. 8). No abdômen, todos os tergos pilosos, exceto por uma estreita margem distal glabra nos TI-TV; superfície dorsal do TI com pilosidade ereta, levemente encurvada para trás, mais fortemente decumbente nas proximidades do bordo distal, as cerdas simples intercaladas com as cerdas plumosas, mais longas nos cantos anteriores e mais curtas em direção ao bordo posterior, no disco chegando a $0,7 \mathrm{~mm}$ de comprimento; tergos II-V exclusivamente com cerdas simples, as mais curtas, grossas e eretas no disco, assemelhando-se a pequenos espinhos, com $0,016 \mathrm{~mm}$ de diâmetro por $0,20 \mathrm{~mm}$ de comprimento, tornando-se mais longas e decumbentes em direção ao bordo distal; tergo VI com as cerdas simples mais longas, com ca. $0,5 \mathrm{~mm}$, eretas, e chegando até o ápice.

Integumento. Na cabeça e tórax, liso, polido, brilhante, apenas os pontos pilígeros, mais densos na fronte, vértice $\mathrm{e}$ porção anterior do mesoscuto (acompanhando a pilosidade mais densa). Na metade inferior da face os pontos muito esparsos e pequenos, apenas alguns pontos mais densos, maiores e alongados para baixo, concentrados na região parocular inferior entre as fóveas clipeais e a margem interna do olho; uma área finamente tesselada acima de cada alvéolo antenal. Nos lados do tórax, a pontuação mais densa e marcada, o integumento praticamente escondido pela pilosidade abundante. Os tergos finamente tesselados, porém ainda brilhantes, os pontos pilígeros bem marcados, acompanhando o maior diâmetro das cerdas, exceto pela faixa marginal glabra, destituída de tais pontos.

Forma e proporções. Cabeça 1,24x mais larga que longa e 1,46x mais larga que a distância clipeocelar. Olhos 2,54x mais longos que largos, divergentes abaixo. Área malar $1,5 \mathrm{x}$ mais longa que o diâmetro do $3^{\circ}$. flagelômero. Comprimento do clípeo 0,61 x sua largura máxima e $0,46 x$ a distância clipeocelar, convexo e mais protuberante basalmente. Mandíbula tão longa quanto $0,65 \mathrm{x}$ a distância clipeocelar, com um pequeno dente mediano. Escapo 1,23x mais longo que a distância alvéoloocelo lateral, seu diâmetro um pouco maior que o do $3^{\circ}$. flagelômero. Distância interocelar $0,76 x$ a ocelorbital e ca. 1,85x o diâmetro do ocelo médio. Vértice achatado, em aclive. 
Distância interalveolar 1,2x maior que o diâmetro do alvéolo antenal e $0,67 \mathrm{x}$ menor que a alveolorbital; a distância alveolocelar lateral 2,58x maior que a interalveolar. Escutelo triangular, elevado no disco, ca. $0,57 \mathrm{x}$ mais curto que largo. Tíbia II 1,48x mais longa que o basitarso II. Tíbia III 2,5x mais longa que larga, e $0,88 \mathrm{x}$ mais longa que a largura máxima da cabeça; canto póstero-distal levemente projetado. Basitarso III $1,67 \mathrm{x}$ mais longo que largo, com o bordo posterior arredondado, aproximadamente em ângulo reto com a margem distal, e o canto póstero-distal levemente arredondado; $1^{\circ}$ tarsômero ca. 1,5x mais longo que largo. Asa anterior 2,97x mais longa que larga e 1,90x mais longa que a largura máxima da cabeça. Bifurcação de $\mathrm{M}+\mathrm{Cu}$ anterior a cu-a. Hámulos, 13. Tergo I com a superfície dorsal mais plana medianamente, sem formar concavidade; o bordo entre a superfície dorsal e a vertical anterior, formando uma dobra mais angulosa que se estende além da região mediana, tornando-se mais arredondada apenas nos cantos.

\section{Macho (Figs. 6, 10, 11, 15, 16; Tab. I)}

Dimensões. Comprimento total aproximado, 11,04 mm; da asa anterior, desde o ápice do esclerito Costal, 9,70 mm (incluindo a tégula, 10,60 mm); largura máxima da cabeça, 4,41 $\mathrm{mm}$; do TII 5,02 mm.

Cor do integumento. Preto, apenas o clípeo, pernas e porção glabra do TII preto-avermelhados; a margem de TI ferrugínea e um pouco translúcida; flagelo preto, o $1^{\circ}$. flagelômero um pouco avermelhado. Membrana das asas hialina, tingida de amarelo, mais fortemente nas células Radial e Marginal; microtríquias amarelas; veias amarelas.

Pilosidade. Preta acinzentada em todo o corpo (na cabeça e tórax, os pêlos um pouco grudados, dificultando a análise); cerdas das peças bucais, tarsômeros de todas as pernas e tíbia III, ferrugíneo-amareladas; lados do propódeo e superfície inferior do escutelo com cerdas plumosas pálidas, a área discal do propódeo, glabra; ápice das cerdas ventrais, pálido. Clípeo, áreas paroculares inferiores e escapo das antenas, praticamente glabros, apenas com finíssimas microcerdas, esparsas, com ca. de $0,05 \mathrm{~mm}$ de comprimento. Pilosidade plumosa, longa e abundante na fronte, vértice e encobrindo densamente o integumento do tórax; na fronte e disco do mesoscuto, mais curtas $(0,44-0,49 \mathrm{~mm})$, no vértice, mesepisternos, cantos anteriores do mesoscuto e no escutelo, muito longa $(0,74-0,88$ $\mathrm{mm}$ ); genas com cerdas simples, eretas não muitos densas e curtas $(0,29 \mathrm{~mm})$. No metassoma, os tergos II-VI com cerdas simples, semi-eretas, grossas e curtas $(0,23 \mathrm{~mm})$, assemelhando-se a espinhos (Fig. 3), mais longas e finas no disco do TII (0,66 mm); algumas cerdas plumosas, mais finas e curtas entremeadas às cerdas simples nos tergos II e III. No TI as cerdas mais finas e ralas, simples e plumosas.

Integumento: Na cabeça e tórax, liso, polido, brilhante (Fig. 6), apenas os pontos pilígeros, mais densos na fronte, vértice e porção anterior do mesoscuto (acompanhando a pilosidade

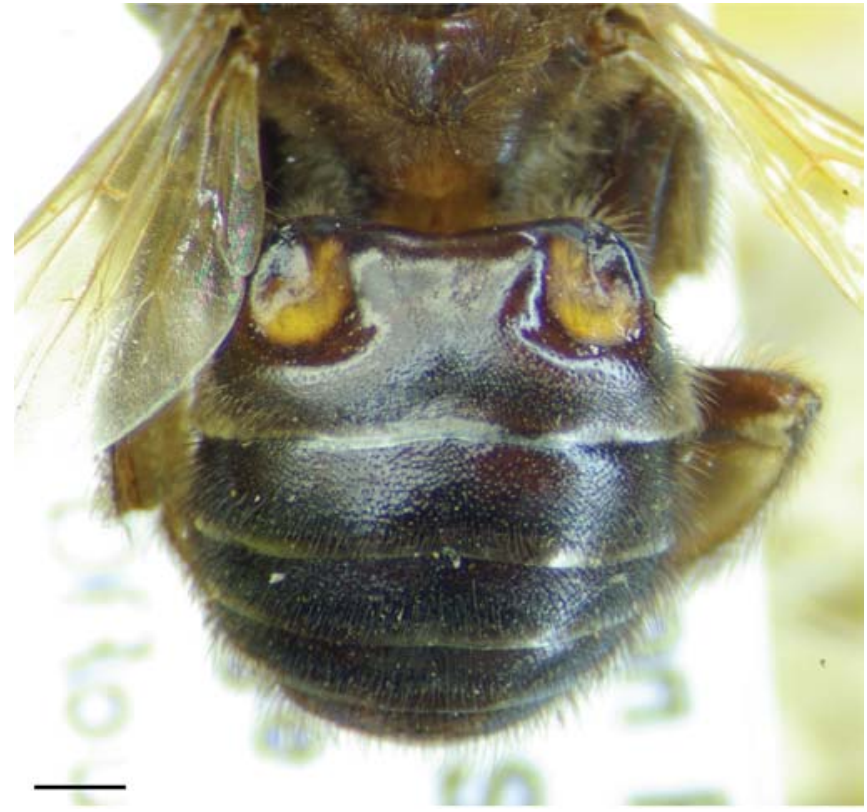

Fig. 23. Abdômen da rainha de Melipona fallax sp. nov. (Portobelo, Colon Prov., Panamá), abertura das glândulas no TI metassomal. Escala $1,0 \mathrm{~mm}$.

mais densa). No clípeo, próximo a margem apical, e na porção superior, algumas áreas com uma micropontuação densa, superficial. Disco do propódeo, liso, polido, brilhante; nos lados, com pontos pilígeros, acompanhando a pilosidade plumosa. No metassoma, o integumento liso, brilhante entre a pontuação pilígera; TI e área glabra basal de TII com um fino tesselado transversal; a pontuação pilígera mais forte e marcada na área pilosa de TII-TVI, acompanhando as cerdas mais grossas; em TII, o limite entre a área pilosa, apical, e a área glabra, basal, marcado por uma linha de pontos maiores e densos, mais esparsos e nítidos para os lados (Fig. 11); a faixa marginal glabra de TI-TV, destituída de pontos.

Forma e proporções. Cabeça 1,23x mais larga que longa e 1,46x mais larga que a distância clipeocelar (Fig. 6). Olhos 2,12x mais longos que largos, subparalelos. Área malar tão longa quanto o diâmetro do $3^{\circ}$ flagelômero. Comprimento do clípeo $0,78 x$ sua largura máxima e $0,54 x$ a distância clipeocelar, saliente (Fig. 6). Mandíbula tão longa quanto metade da distância clipeocelar, o ápice inerme, sinuoso. Escapo 1,29x mais longo que a distância alvéolo-ocelo lateral, seu diâmetro máximo (próximo ao ápice), 1,75x o do $3^{\circ}$ flagelômero. Distância interocelar $0,78 \mathrm{x}$ a ocelorbital e ca. 1,44x o diâmetro do ocelo médio. Vértice formando uma superfície achatada, em aclive em direção ao topo da cabeça, um pouco elevado atrás dos ocelos. Distância interalveolar 1,22x maior que o diâmetro do alvéolo antenal e igual a alveolorbital; a distância alveolocelar lateral 2,18x maior que a interalveolar; distância entre o ocelo médio e o lateral, ca. 0,28x o diâmetro do ocelo médio (Fig. 10). Escutelo triangular, elevado no disco, ca. 0,56x mais curto que largo. Tíbia III 3,08x mais longa que larga, e 0,82x a largura máxima da cabeça. Basitarso III 2,43x mais longo que largo, 
Tabela I. Medidas (mm): M. fallax sp. nov. - holótipo, operária (Portobelo, Colon, Panamá), parátipo, macho (Panamá, Chepo), parátipo, rainha (Portobelo, Colon, Panamá); M. titania - macho (Peru, Napo river), operária (Pastaza, Puyo, Ecuador); M. fuliginosa - macho, rainha (Tapera, lago Caurés, rio Negro, AM, Brasil), operária (Kourou, $25 \mathrm{Km} \mathrm{SW}$, Guiana Francesa).

\begin{tabular}{|c|c|c|c|c|c|c|c|c|}
\hline \multirow[b]{2}{*}{ Medidas } & \multicolumn{3}{|c|}{ M. fallax sp. nov. } & \multicolumn{3}{|c|}{ M. fuliginosa } & \multicolumn{2}{|c|}{ M. titania } \\
\hline & $\begin{array}{l}\text { operária } \\
\text { holótipo }\end{array}$ & $\begin{array}{l}\text { macho } \\
\text { parátipo }\end{array}$ & $\begin{array}{c}\text { rainha } \\
\text { parátipo }\end{array}$ & macho & operária & rainha & macho & operária \\
\hline Comprimento total aproximado & 11,70 & 11,04 & 11,17 & 12,76 & 11,29 & 13,74 & 12,39 & 11,90 \\
\hline Largura máxima da cabeça & 4,85 & 4,41 & 4,19 & 4,58 & 4,95 & 4,36 & 4,61 & 4,85 \\
\hline Comprimento da cabeça & 3,92 & 3,58 & 3,48 & 3,87 & 4,07 & 3,58 & 3,87 & 3,97 \\
\hline Comprimento do olho & 2,99 & 2,60 & 2,38 & 2,79 & 3,04 & 2,45 & 2,77 & 2,89 \\
\hline Largura do olho & 1,18 & 1,23 & 0,88 & 1,23 & 1,18 & 0,88 & 1,32 & 1,18 \\
\hline Distância interorbital superior & 2,79 & 2,40 & 2,55 & 2,62 & 2,84 & 2,70 & 2,60 & 2,84 \\
\hline Distância interorbital máxima & 3,19 & 2.52 & 2,99 & 2,70 & 3,23 & 2,99 & 2,70 & 3,19 \\
\hline Distância interorbital inferior & 3,04 & 2,35 & 2,89 & 2,50 & 3,11 & 2,94 & 2,43 & 3,06 \\
\hline Diâmetro do ocelo médio & 0,33 & 0,36 & 0,28 & 0,36 & 0,33 & 0,30 & 0,39 & 0,36 \\
\hline Distância entre o ocelo médio e o lateral & 0,16 & 0,10 & 0,16 & 0,16 & 0,19 & 0,15 & 0,15 & 0,16 \\
\hline Distância interocelar (ocelos laterais) & 0,61 & 0,52 & 0,61 & 0,64 & 0,64 & 0,54 & 0,64 & 0,64 \\
\hline Distância ocelorbital (ocelo lateral) & 0,81 & 0,66 & 0,76 & 0,69 & 0,78 & 0,83 & 0,69 & 0,78 \\
\hline Distância interalveolar & 0,59 & 0,54 & 0,64 & 0,54 & 0,64 & 0,59 & 0,56 & 0,64 \\
\hline Distância alveolorbital & 0,88 & 0,54 & 0,81 & 0,59 & 0,83 & 0,78 & 0,54 & 0,78 \\
\hline Distância alveolocelar (ocelo lateral) & 1,52 & 1,18 & 0,86 & 1,27 & 1,47 & 1,32 & 1,37 & 1,57 \\
\hline Diâmetro do alvéolo & 0,49 & 0,44 & 0,44 & 0,49 & 0,49 & 0,44 & 0,51 & 0,49 \\
\hline Comprimento do clípeo (meio) & 1,54 & 1,62 & 1,35 & 1,67 & 1,57 & 1,37 & 1,67 & 1,49 \\
\hline Largura máxima do clípeo & 2,55 & 2,08 & 2,25 & 2,43 & 2,70 & 2,33 & 2,21 & 2.45 \\
\hline Larg. do clípeo nas fóveas tentoriais & 1,47 & 1,32 & 1,42 & 1,37 & 1,42 & 1,37 & 1,37 & 1,45 \\
\hline Distância clipeocelar (ocelo médio) & 3,33 & 3,01 & 2,94 & 3,14 & 3,28 & 2,94 & 3,23 & 3,33 \\
\hline Comprimento da área malar & 0,29 & 0,20 & 0,44 & 0,15 & 0,29 & 0,44 & 0,20 & 0,34 \\
\hline Comprimento do escapo & 1,86 & 1,52 & 1,86 & 1,62 & 1,86 & 1,96 & 1,57 & 1,86 \\
\hline Diâm. escapo (máximo) & 0,25 & 0,34 & 0,25 & 0,34 & 0,25 & 0,25 & 0,34 & 0,27 \\
\hline Diâmetro 3으. & 0,20 & 0,20 & 0,20 & 0,25 & 0,20 & 0,22 & 0,27 & 0,25 \\
\hline Comprimento do pedicelo + flagelo & 3,43 & 4,26 & 4,02 & 4,36 & 3,68 & 4,36 & 4,17 & 3,53 \\
\hline Comprimento do $1^{\circ} \mathrm{fl}$ & 0,37 & 0,25 & 0,29 & 0,25 & 0,37 & 0,29 & 0,22 & 0,34 \\
\hline Comprimento do $2^{\circ} \mathrm{fl}$. & 0,32 & 0,34 & 0,39 & 0,39 & 0,34 & 0,39 & 0,39 & 0,29 \\
\hline Comprimento do $3^{\circ} \mathrm{fl}$. & 0,32 & 0,37 & 0,39 & 0,42 & 0,34 & 0,37 & 0,37 & 0,32 \\
\hline Comprimento da mandíbula & 2,18 & 1,52 & 1,72 & 1,62 & 2,25 & 1,89 & 1,62 & 2,21 \\
\hline $\begin{array}{l}\text { Comprimento da asa anterior (do ápice do } \\
\text { esclerito Costal) }\end{array}$ & 9,20 & 9,70 & 7,90 & 9,60 & 9,20 & 8,00 & 9,40 & 8,70 \\
\hline $\begin{array}{l}\text { Comprimento da asa anterior (do ápice do } \\
\text { esclerito Costal + tégula) }\end{array}$ & 10,40 & 10,60 & 8,90 & 10,60 & 10,30 & 8,90 & 10,40 & 10,0 \\
\hline Largura da asa anterior & 3,10 & 3,20 & 2,70 & 3,20 & 3,30 & 2,70 & 3,00 & 3,20 \\
\hline Largura do mesoscuto & 3,43 & 3,53 & 3,48 & 3,92 & 3,68 & 3,33 & 3,53 & 3,43 \\
\hline Comprimento do mesoscuto & 3,33 & 2,94 & 2,55 & 3,33 & 3,14 & 2,60 & 3,04 & 3,04 \\
\hline Largura do escutelo & 2,25 & 2,11 & 1,86 & 2,30 & 2,25 & 1,72 & 2,16 & 2,21 \\
\hline Comprimento do escutelo & 1,27 & 1,18 & 1,18 & 1,42 & 1,27 & 1,27 & 1,27 & 1,23 \\
\hline Comprimento da tíbia III & 4,29 & 3,63 & 3,77 & 3,77 & 4,26 & 4,02 & 3,63 & 4,19 \\
\hline Largura máxima da tíbia III & 1,72 & 1,18 & 1,32 & 1,32 & 1,67 & 1,35 & 1,27 & 1,72 \\
\hline Comprimento do basitarso III & 1,76 & 1,67 & 1,96 & 1,86 & 1,91 & 2,06 & 1,72 & 1,96 \\
\hline Largura do basitarso III & 1,05 & 0,69 & 0,59 & 0,83 & 1,13 & 0,69 & 0,83 & 1,13 \\
\hline Largura do TII & 5,29 & 5,02 & 5,24 & 5,59 & 5,44 & 5,73 & 5,59 & - \\
\hline Compr. cerdas do escapo & 0,15 & 0,05 & 0,20 & - & 0,12 & 0,20 & 0,05 & 0,25 \\
\hline Compr. cerdas do clípeo & 0,22 & - & 0,34 & - & 0,29 & 0,25 & - & 0,29 \\
\hline Compr. cerdas do vértice & 0,88 & 0,98 & 0,88 & 0,88 & 0,83 & 0,93 & 0,78 & 0,98 \\
\hline Compr. cerdas do disco do mesoscuto & 0,59 & 0,54 & 0,49 & 0,49 & $?$ & 0,44 & 0,54 & 0,69 \\
\hline Compr. cerdas na porção anterior do mesoscuto & 0,98 & 0,74 & 0,83 & 0,74 & 1,18 & 0,88 & 0,81 & $?$ \\
\hline Comprimento das cerdas do escutelo & 1,10 & 0,88 & 0,98 & 0,74 & 1,13 & 0,88 & 0,93 & 1,03 \\
\hline
\end{tabular}

subretangular, levemente estreitado para a base (Fig. 15); o $1^{\circ}$ tarsômero tão longo quanto largo, o canto póstero-distal apenas moderadamente projetado (Fig. 15). Asa anterior, 3,03x mais longa que larga e 1,08x mais longa que a largura máxima da cabeça. Bifurcação de $\mathrm{M}+\mathrm{Cu}$ anterior a cu-a. Hámulos, 1413. Genitália conforme Fig. 16; os esternos pré-genitais VII e VIII como em M. fuliginosa (Figs. 20, 21). 
Rainha (Fig. 23, Tab. I; do Panamá, Colón Prov., Portobelo 12 km SW - Santa Rita Ridge 20, 12.I.1988, D. Roubik leg., rainha virgem). Cor e textura do integumento como nas operárias, avermelhado na parte ventral e pernas. Pilosidade preta-pardacenta, plumosa na fronte e tórax; metade inferior da face lisa, brilhante, com cerdinhas eretas, esparsas, no clípeo. O tergo I extensamente glabro na parte anterior, as cerdas simples, eretas, restritas a uma faixa apical, intercaladas, nos lados, com algumas cerdas plumosas. No tergo II e seguintes, a pilosidade ereta, simples, relativamente esparsa, como nas operárias. A larga depressão a cada lado do tergo I (Fig. 23), como em M. fuliginosa e M. titania (ver comentários em M. titania, item "Discussão"). As genas pouco protuberantes em baixo, como em $M$. titania. As medidas estão relacionadas na Tab. I. Dentre o material examinado há uma rainha de "Colômbia, Ant., Rio Porce", que difere daquela do Panamá, por apresentar o primeiro tergo metassomático bem mais longo (2,4 mm de comprimento no exemplar do Panamá, e 2,7 mm no exemplar da Colômbia); nos demais caracteres, aparentemente não difere.

Material-tipo. Holótipo, operária, de 'Rood [sic = Road ?], Km 20. \IV-85 Roubik 850829', 'Colon Prov. Panamá \ Portobelo, 15 Km SW । "Santa Rita Ridge", depositado no STRI, e mais os seguintes parátipos: 5 operárias com etiqueta de procedência igual a do holótipo, nos. 850827828, 850830-832, depositadas na RPSP; 1 rainha virgem, 9 machos e 15 operárias de ninho, de PANAMA: Panama Prov. Chepo, ca. $13 \mathrm{~km}$ NE; El Llano-Cartí Road, 31 Mar 1982, coll. \#40, 44, 45, 46, 52, 54 , 61 (1981-83), s/n (1989), D. Roubik leg., depositados no STRI; 1 macho, de PANAMA, Pan. Prov., Chepo 25 km E, mar. 1989 (?) D. Roubik coll. Nest, depositado na RPSP; 4 rainhas virgens e 234 operárias de ninho, de PANAMA: Colón Prov. Santa Rita Ridge (ca. 12 km SW Portobelo, or $25 \mathrm{~km}$ NE Puerto Pilon) 400-500 m elevation, 10 Feb. 1981, 20 Oct 1982, 22 Apr. 1985, \& coll. \# 10, 28, 31, 42, 46, 84, 86 (1980-1989), D. Roubik leg.; uma rainha virgem de "PANAMA; Colon Prov \Portobelo $12 \mathrm{~km} \mathrm{SW} \mathrm{\ Santa} \mathrm{Rita} \mathrm{Ridge} \mathrm{20 \backslash 12/1/1988} \mathrm{D.}$ Roubik", e duas operárias de "PANAMA: Darien Prov. \Cana Field Sta. $450 \mathrm{~m} \backslash 6$ Feb 2000 D Roubik" depositados no STRI, USNM e D. Fairchild Entomological Collection, University of Panamá.

Material adicional examinado. COSTA RICA. San Jose: Cerro Nara, 28.VIII.1968, Wille \& Orozco leg. (6 ops., RPSP); Pozo Azul (unión rios Parrita y Candelária), 4.XII.1961, A. Wille leg. (3 ops., RPSP); Cartago: Turrialba - CATIE, 12-13.IX.1998, D. Roubik leg., on Bixa (1 op., STRI); Limón: Pandora, 23.VIII.1963, 2000ft., Wille, Kerfoot C. D. \& D. R. Michener leg. (1 op., RPSP). PANAMA. Bocas del Toro: Miramar, 29.VIII.1980, D. Roubik leg., coll. \#12 (1 op., STRI); Chiriquí: Fortuna, $1100 \mathrm{~m}$ elev., 24.V.1985, D. Roubik leg., s/n (2 ops., STRI); Veraguas: Santa Fé, Cerro Tute, 900 m elev., 9.IX.1982, D. Roubik leg., s/n (7 ops., STRI); Coclé: El Copé, 8 km N, 31.VIII.1988, D. Roubik leg., s/n (3 ops., STRI); Panamá: Barro Colorado Island, 2.V.1979, D. Roubik leg., coll \# 21, on flowers of Hybanthus prunifolius (1 op., STRI); Cerro Campana Nat. Pk., 850 m elev., 1980-1983, D. Roubik leg., coll. \#4, 19, 28, 45 (5 ops., STRI); ibidem, 7.V.1981, R. W. Brooks leg., on flowers of Conostegia xalapensis (1 op., STRI); Chepo, 13 km NE, El Llano Cartí Road, 4.XI.1982, D. Roubik leg., on Bixa orellana (1 opéraria anã, STRI); Gamboa, $8 \mathrm{mi} \mathrm{N}, 21 . X I .1979$, D. Roubik leg., coll \# 19 (3 ops., STRI); ibidem, idem, Pipeline Road, 1980, 1984 (coll. \# 34, 128 (2 ops., STRI); ibidem, idem, 8.XII.1982, s/n (6 ops., STRI); Summit Gardens, 2 km N, 7.IV.1983, D. Roubik leg, $\mathrm{s} / \mathrm{n}$, on flowers of Miconia argentea (1 op., STRI); San Blas: Kuna Yala, Puerto Obaldía, 1979, 1981, D. Roubik leg. coll. \#10, 12 (6 ops., STRI); Darién: Canglón, 14.XI.1982, D. Roubik leg., coll. \# 17 (7 ops., STRI); Chepo, 212 km E, 24.IX.1984, D. Roubik leg., coll. \#16 (1 op., STRI); COLÔMBIA. Antioquia: Rio Porce, 2.IV.1968, R. L. Dressler (1 rainha, DZUP); EQUADOR. Esmeraldas: Quinindé, VI-86, G. Onore leg. (1 op., RPSP).
Distribuição geográfica e habitat. Ocorre na América Central, desde a Nicarágua (Schwarz 1934), até o Panamá, e pela costa Pacífica, chega até Guayaquil, no Equador (Cockerell 1919); na Costa Rica ocorre tanto na região do Atlântico como na do Pacífico (Wille 1976; Biesmeijer 1997) (Fig. 22). É possível que $M$. fallax sp. nov. também esteja presente nos vales do Cauca e Magdalena (Nates-Parra 1983, 2001), mas exemplares dessas regiões não foram examinados.

Nidificação e outras notas bionômicas. Ninhos desta espécie, popularmente conhecida como "Tobé" no Panamá (Roubik 1981), foram descritos e ilustrados por Wille \& Michener (1973), Wille (1976), Roubik (1981, 1983, 1989) e Coloma (1986). Os ninhos não diferem daquele de Melipona fuliginosa, são construídos em ocos de árvores vivas, e a entrada constituída de um tubo, composto de resinas e sementes. Roubik (1989: 28, 29 fig. 2.1; Roubik in litt. 2008) menciona ter observado, no Panamá, onde estudou 12 ninhos, que $M$. fallax sp. nov. (citada como M. fuliginosa) coleta e deposita, continuamente, no tubo de entrada, sementes e resinas alaranjadas de Vismia (Clusiaceae - provavelmente sementes de Coussapoa e não de Vismia, conforme o próprio Roubik in litt. 2008). Coloma (1986: 36, 37, 113), entretanto, refere-se a um ninho estudado em La Sexta, Esmeralda, Equador, em um tronco caído de Ficus sp., como tendo a estrutura de entrada lisa, feita apenas de terra e própoles e o batume crivado de poros cilíndricos, com "salpicaciones amarillentas y rojizas" [provavelmente sementes]. Roubik (1981) encontrou no Panamá, uma colônia mista natural, em que M. fallax sp. nov. e M. (Michmelia) panamica Cockerell, 1912 (citada como M. fasciata) coexistiam em uma mesma cavidade de árvore, compartilhando inclusive a mesma câmara de cria; ele relata ainda que nesse ninho não havia batume superior e o inferior não era perfurado. Segundo Coloma (1986), no Equador, essas abelhas são agressivas e agitadas. Roubik (in litt. 2008) relata que no Panamá, Melipona fallax sp. nov. é uma das poucas espécies de Meliponini que ataca ativamente ninhos de Apis mellifera scutellata Lepeletier, 1836, na floresta, decapitando as operárias defensoras quando estão realizando pilhagem de mel. Roubik (2000) refere-se à Melipona fallax $\mathbf{s p . ~}$ nov. (citada como M. fuliginosa) como polinizador da orquídea Maxillaria na Costa Rica e Panamá.

Etimologia. Do latim, fallax = enganador, que induz a erro.

Nomes populares: jicote-congo (Costa Rica), tobé (Panamá).

Discussão. Camargo \& Pedro (2007: 399) já haviam chamado a atenção para a existência desta espécie, aqui descrita como $M$. fallax sp. nov. Distingue-se de M. fuliginosa, pela ausência de cerdas plumosas nos tergos II-V, área parocular inferior plana, forma do basitarso e $1^{\circ}$ tarsômero da perna III. De M. titania, separa-se principalmente pela forma do penicilo das operárias; no macho, os olhos são paralelos, e os pontos fortemente marcados separando a área pilosa da área basal glabra no TII, além do fato de estar restrita ao lado ocidental dos Andes. 
Comentários gerais sobre o padrão biogeográfico. $\mathrm{O}$ padrão de distribuição / vicariância das espécies do grupo fuliginosa, coincide, em certa extensão, com os traçados e componentes biogeográficos postulados a partir de estudos com outros táxons de Meliponini (Camargo \& Moure 1996; Camargo 1996; Camargo \& Pedro 2003; Pedro \& Camargo 2003) e outros grupos animais (Amorim \& Pires 1996), sugerindo, ao menos em parte, uma história em comum.

Cinco componentes biogeográficos, subordinados a dois megacompartimentos, ou traçados, foram identificados por esses autores para a região Neotropical (s. str.): traçado NW, com os componentes Chocó - América Central (Chocó-CA), sudoeste da Amazônia (SWAm), norte da Amazônia (NAm), e traçado SE, com os componentes Sul dos rios Madeira/ Amazonas até o noroeste da Argentina (SEAm) e Mata Atlântica do sudeste do Brasil (Att.). Os cladogramas de área resultantes e o cenário biogeográfico para Meliponini e outros táxons podem ser vistos em Camargo (1996, 2006), Camargo \& Pedro (2003) e Amorim \& Pires (1996).

Melipona fallax sp. nov. ocupa o componente Chocó-CA, enquanto $M$. titania ocupa a área irmã SWAm, sugerindo que não só podem ser espécies irmãs, como são de idade geológica relativamente recente, correspondendo aos ramos fulvohirtaleucogastra do gênero Geotrigona e bilineata-epiphytophila do gênero Partamona (a quebra que origina os componentes Chocó-CA e SWAm, possivelmente está relacionada com a orogenia dos Andes equatorianos, que atingem cotas superiores a 3.000-4.000 m no Plio-Pleistoceno; Camargo \& Pedro 2003, Camargo 2006).

Melipona fuliginosa tem uma larga distribuição, ocupando o componente NAm, no traçado NW, e os componentes SEAm (até o noroeste da Argentina) e Atl., no traçado SE. Se realmente se trata de uma única espécie, como admitido nesse trabalho, esse padrão é inusitado - é a única espécie de Meliponini com tal distribuição - , ou não respondeu a nenhum dos eventos de vicariância correspondentes aos grandes traçados e componentes, e nem, subsequentemente, aos eventos de fragmentação interna de cada componente, ou sua distribuição / dispersão é posterior a esses eventos. A distribuição de $M$. fuliginosa fica ainda mais estranha quando comparada a de M. titania.

Pelos registros disponíveis, $M$. titania está restrita ao componente SWAm, o único componente onde $M$. fuliginosa não ocorre (o registro mais a oeste que se tem, é de Xapuri, Acre). Essa exclusão pode estar relacionada com causas ecológicas, ou mesmo com as barreiras biogeográficas, que deram origem ao componente SWAm, e que teriam permanecido até mais recentemente. Não é o que acontece, entretanto, com alguns outros grupos de Meliponini, onde algumas espécies, principalmente as relacionadas ao componente NAm, invadem grandemente o SWAm, sobrepondo-se aos grupos irmãos endêmicos desse componente (e.g., espécies de Geotrigona, Camargo \& Moure 1996). De qualquer forma, com os conhecimentos atuais, cabe aqui apenas registrar os fatos, não há como hipotetizar uma história plausível. Pouco se sabe da biologia das espécies desse grupo, especialmente de $M$. fuliginosa, que apesar de sua larga distribuição, seus ninhos são muito raros, talvez muito distantes uns dos outros, possivelmente relictos biogeográficos.

Também, não se sabe ainda se o grupo fuliginosa ocupa uma posição basal ou derivada em relação aos demais subgrupos do subgênero Michmelia, todavia, pela congruência, ao menos em parte, em relação aos componentes biogeográficos, pode-se admitir a hipótese de que, embora com apenas três espécies, seria, no mínimo, de idade equivalente aos subgrupos, muito mais diversificados, de Geotrigona, Paratrigona e Partamona, que habitam o traçado NW (Camargo \& Moure 1996, Camargo 1996, Camargo \& Pedro 2003). Dentre os demais Meliponini estudados, encontra paralelo somente em Ptilotrigona, outra réplica biogeográfica e também com apenas três espécies (Camargo \& Pedro 2004), entretanto, Ptilotrigona lurida (Smith, 1854) não chega tão ao sul quanto Melipona fuliginosa.

Agradecimentos. A Claus Rasmussen, pelas fotos de ninho de $M$. titania (inclusive a Fig. 2), informações sobre ninhos e exemplares do Peru; ao Dr. David W. Roubik (STRI), pelo envio do material do Panamá, inclusive o exemplar designado como holótipo de $M$. fallax sp. nov. e informações sobre comportamento; ao Dr. J. G. Rozen (AMNH), pelo envio do macho de $M$. titania estudado por Schwarz; ao Dr. Gabriel A. R. Melo pelo envio do material depositado no DZUP; a Rodrigo Gonçalves, que examinou os exemplares depositados no MZUSP e transcreveu todos os dados das etiquetas; a Rodrigo Silva pelas fotos (Figs. 3-4, 8-9) no Microscópio de varredura do Depto. de Química FFCLRP, USP; aos revisores anônimos, pelas correções e sugestões.

\section{REFERÊNCIAS}

Amorim, D. S. \& M. R. S. Pires. 1996. Neotropical biogeography and a method for maximum biodiversity estimation, p. 183-219. In: C. E. M. Bicudo \& N. A. Menezes (eds). Biodiversity in Brazil, a first approach. São Paulo, CNPq, 326 p.

Barcelar-Lima, C. G.; D. C. B. Freire; A. Coletto-Silva; K. B. Costa; J. P. B. Laray; H. C. Vilas-Boas \& G. A. Carvalho-Zilse. 2006 Melitocoria de Zygia racemosa (Ducke) Barneby \& Grimes por Melipona seminigra merrillae Cockerell, 1919 y Melipona compressipes manaosensis Schwarz, 1932 (Hymenoptera, Meliponina) en la Amazonía Central, Brasil. Acta Amazonica 36: 343-348.

Benoist, R. 1933. Description d'une nouvelle espéce de Mélipone (Hym. Apidae) de la République de l'Équateur. Bulletin de la Societé Entomologique de France 38: 52.

Biesmeijer, J. C. 1997. The organisation of foraging in stingless bees of the genus Melipona: an individual-oriented approach. Utrecht, Elinkwijk bv, 263 p.

Brown, J. C. \& C. Albrecht. 2001. The effect of tropical deforestation on stingless bees of the genus Melipona (Insecta: Hymenoptera: Apidae: Meliponini) in central Rondonia, Brazil. Journal of Biogeography 28: 623-634.

Bruijn, L. L. M. \& M. J. Sommeijer. 1997. Colony foraging in different species of stingless bees (Apidae, Meliponinae) and the regulation of individual nectar foraging. Insectes Sociaux 44: 35-47.

Camargo, J. M. F. 1988. Meliponinae (Hymenoptera, Apidae) da coleção do "Istituto di Entomologia Agraria", Portici, Itália. Revista Brasileira de Entomologia 32: 351-374.

Camargo, J. M. F. 1994. Biogeografia de Meliponini (Hymenoptera, Apidae, Apinae): a fauna amazônica, p. 46-59. In: R. Zucchi, P. M Drumond, P. G. Fernandes-da-Silva \& S. C. Augusto (eds.). Anais do I Encontro Sobre Abelhas. Ribeirão Preto: Faculdade de Filosofia, Ciências e Letras de Ribeirão Preto, Universidade de São Paulo, $\mathrm{xx}+308 \mathrm{p}$. 
Camargo, J. M. F. 1996. Meliponini neotropicais (Apinae, Apidae, Hymenoptera): biogeografia histórica, p. 107-121. In: C. A. Garófalo et al (comissão organizadora), Anais do II Encontro sobre Abelhas de Ribeirão Preto. Ribeirão Preto, Universidade de São Paulo, Faculdade de Filosofia, Ciências e Letras de Ribeirão Preto, xxii+351 p.

Camargo, J. M. F. 2006. A Amazônia não é uma unidade histórica!, p. 47-49. In: W. C. Santana, C. H. Lobo, K. H. Hartfelder et al. (eds), Anais do VII Encontro sobre Abelhas, Ribeirão Preto, FFCLRPUSP, FMRP-USP, publicação eletrônica em mídia digital (CDROM), $850 \mathrm{p}$.

Camargo, J. M. F. \& J. S. Moure. 1996. Meliponini Neotropicais: o gênero Geotrigona Moure, 1943 (Apinae, Apidae, Hymenoptera), com especial referência à filogenia e biogeografia. Arquivos de Zoologia 33: 95-161.

Camargo, J. M. F. \& S. R. M. Pedro. 2003. Meliponini Neotropicais: o gênero Partamona Schwarz, 1939 (Hymenoptera, Apidae, Apinae) - bionomia e biogeografia. Revista Brasileira de Entomologia 47: 311-372.

Camargo, J. M. F. \& S. R. M. Pedro. 2004. Meliponini neotropicais: o gênero Ptilotrigona Moure (Hymenotera, Apidae, Apinae). Revista Brasileira de Entomologia 48: 353-377.

Camargo, J. M. F. \& S. R. M. Pedro. 2005. Meliponini Neotropicais: o gênero Dolichotrigona Moure (Hymenoptera, Apidae, Apinae). Revista Brasileira de Entomologia 49: 69-92.

Camargo J. M. F. \& S. R. M. Pedro. 2007. Meliponini Lepeletier, 1836, p. 272-578. In: Moure, J. S., Urban, D. \& Melo, G. A. R. (Orgs.). Catalogue of Bees (Hymenoptera, Apoidea) in the Neotropical Region. Curitiba, Sociedade Brasileira de Entomologia, xiv +1058 p.

Camargo, J. M. F.; J. S. Moure \& D. W. Roubik. 1988. Melipona yucatanica new species (Hymenoptera: Apidae: Meliponinae); stingless bee dispersal across the Caribbean arc and post-Eocene vicariance. The Pan-Pacific Entomologist 64: 147-157.

Castello Branco, L. S. D. 1845. Memoria á cerca das abelhas da Provincia do Piauhy no Imperio do Brasil. O Auxiliador da Indústria Nacional 13: 49-72.

Cockerell, T. D. A. 1919. Bees in the collection of the United States Museum. - 3. Proceedings of the United States National Museum 55: 167-221

Coloma, L. A. 1986. Contribución para el conocimiento de las abejas sin aguijón (Meliponinae, Apidae, Hymenoptera) de Equador. Monografia de Licenciatura em Ciências Biológias, Pontificia Universidad Catolica del Equador, Quito, Equador, 146p.

Cortopassi-Laurino, M. 2004. A Uruçu Boi da Amazônia, Melipona fuliginosa Lepeletier, 1836. Mensagem Doce 77: 1-3 (Mensagem Doce Online: http:// www.apacame.org.br/mensagemdoce/77/relato/ $\mathrm{htm})$.

Cortopassi-Laurino, M. 2005. A abelha jataí: uma espécie bandeira? (Tetragonisca angustula Latreille, 1881). Mensagem Doce 80: $34-38$.

Crane, E. 1999. The world history of beekeeping and honey hunting. New York, Routledge xxii+682 p.

Cunha, R. A. 1973. Taxonomia numérica de alguns Meliponinae (Hymenoptera- Apidae). Ciência Biológica (Portugal) 1: 2542.

Cunha, R. A. 1991. Revisão da taxonomia de alguns Meliponinae por métodos fenéticos (Hym., Apidae). Naturalia (São Paulo) 16: $33-53$.

Dalla Torre, C. G. de 1896. Catalogus Hymenopterorum; Hucusque Descriptorum Systematicus et Synonymicus. Leipzig, Engelmann Vol. 10, viii+643 p.

Darchen, R. 1969. La biologie des Trigones et des Mélipones (Hyménoptéres Apidae) a la lumbre des travaux récents. Année Biologique 8: 455-490.

Dodson, C. H. 1967. Relationships between pollinators and orchid flowers, p. 1-72. In: H. Lent (ed.). Atas do simpósio sôbre a biota amazônica. Rio de Janeiro, Conselho Nacional de Pesquisas, Vol. 5: zoologia, $603 \mathrm{p}$

Dominique, J. 1898. Coup d'oeil sur les Mellifères Sud- Américains du Muséum de Nantes. Bulletin de la Societé des Sciences Naturelles de l'Ouest de la France (Nantes) 8: 57-65.

Ducke, A. 1901. Beobachtungen über Blütenbesuch, Erscheinungszeit etc. der bei Pará vorkommenden Bienen. Zeitschrift für systematische Hymenopterologie und Dipterologie 1: 49-67.

Ducke, A. 1902 a. Beobachtungen über Blütenbesuch, Erscheinungszeit etc. der bei Pará vorkommenden Bienen. Allgemeine Zeitschrift für Entomolologie 7: 417-422.

Ducke, A. 1902 b. Die stachellosen Bienen (Melipona Ill.) von Pará, nach dem Materiale der Sammlung des Museu Goeldi beschrieben. Zoologische Jahrbücher Abteilung für Systematik, Geographie und Biologie der Tiere 17: 285-328.

Ducke, A. 1910. Zur Synonymie der neotropischen Apidae. (Hym.). Deutsche Entomologische Zeitschrift 6: $362-369$.

Ducke, A. 1916. Enumeração dos Hymenopteros colligidos pela Comissão e Revisão das espécies de abelhas do Brasil. Commissão de Linhas Telegraphicas Estrategicas de Matto Grosso ao Amazonas 35, anexo $\mathrm{N}^{\text {o. }}$ 5, História Natural, Zologia: 3-171.

Ducke, A. 1925. Die stachellosen Bienen (Melipona) Brasiliens, Nach morphologischen und ethologischen Merkmalen geordnet. Zoologische Jahrbücher. Abteilung für Systematik, Geographie und Biologie der Tiere 49: 335-448.

Ducke, A. 1945. Himenópteros, Enumeração dos espécimes coligidos pela Comissão e Revisão das Espécies de Abelhas do Brasil. Comissão de Linhas Telegráficas e Estratégicas de MatoGrosso ao Amazonas ("Comissão Rondon"), publicação no. 35, anexo no. 5 de Zoologia. Rio de Janeiro, Ministério da Agricultura, Conselho Nacional de Proteção aos Índios, $2^{\mathrm{a}}$. ed, 137 p.

Engel, M. S. \& F. Dingemans-Bakels. 1980. Nectar and pollen resources for stingless bees (Meliponinae, Hymenoptera) in Surinam (South America). Apidologie 11: 341-350.

Fernandes-Salomão, T. M.; J. I. Muro-Abad; L. A. O. Campos \& E. F. Araújo. 2002. Mitochondrial and nuclear DNA characterization in the Melipona species (Hymenoptera, Meliponini) by RFLP analysis. Hereditas (Lund) 137: 229-233.

Garcia, M. V. B.; M. L. Oliveira \& L. A. O. Campos. 1992. Use of seeds of Coussapoa asperifolia magnifolia (Cecropiaceae) by stingless bees in the Central Amazonian Forest (Hymenoptera: Apidae: Meliponinae). Entomologia Generalis 17: 255-258.

Gonzalez-B., V. H. \& R. Ospina-Torres. 2000. Eufriesea nigrescens y E. pretiosa (Hymenoptera: Apidae: Euglossini): un caso de oportunismo o simbiosis? Caldasia 22: 357-359.

Gribodo, G. 1893. Note imenotterologiche. Nota. II. Nuovi generi e nuove specie di Imenotteri Antofili ed osservazioni sopra alcune specie giá conosciute. Bollettino dela Società Entomologica Italiana 25: 248-287.

Griswold, T.; F. D. Parker \& P. E. Hanson. 1995. The bees (Apidae), p. 650-691. In: P. E. Hanson \& I. D. Gauld (eds). The Hymenoptera of Costa Rica. London, Natural History Museum, 893 p.

Guerino, A. C. \& V. T. P. Oliveira. 2002. Glândulas tegumentares do abdômen, p. 111-126. In: C. Cruz-Landim \& F. C. Abdalla (eds.) Glândulas exócrinas das abelhas. Ribeirão Preto, FUNPEC$\mathrm{RP}, \mathrm{xi}+181 \mathrm{p}$.

Heard, T. A. 1999. The role of stingless bees in crop pollination. Annual Review of Entomology 44: 183-206.

Ihering, H. 1903. Biologie der stachellosen Honigbienen Brasiliens. Zoologische Jahrbücher. Abteilung für Systematik, Geographie und Biologie der Tiere 19: 179-287.

Ihering, H. 1930. Biologia das abelhas melliferas do Brasil. Boletim de Agricultura 31: 435-506, 649-714.

Ihering, R. 2002. Dicionário dos animais do Brasil. Rio de Janeiro, DIFEL, 588 p. + 12 pranchas.

Kerr, W. E. 1948. Estudos sobre o gênero Melipona. Anais da Escola Superior de Agricultura "Luiz de Queiroz" 5: 181-276 + 51 figuras.

Kerr, W. E. 1951. Bases para o estudo da genética de populações dos Hymenoptera em geral e dos Apinae sociais em particular. Anais da Escola Superior de Agricultura "Luiz de Queiroz" 8: 219-354

Kerr, W. E. 1969. Some aspects of the evolution of social bees (Apidae). Evolutionary Biology 3: 119-175.

Kerr, W. E. 2002. Extinção de espécies: a grande crise biológica do momento e como afeta os meliponínios, p. 4-9. In: Garófalo et al. (ed.). V Encontro sobre abelhas: anais. Ribeirão Preto, Faculdade de Filosofia, Ciências e Letras de Ribeirão Preto, Universidade de São Paulo, Departamento de Biologia, xxv+355 p.

Kerr, W. E. \& H. Esch. 1965. Comunicação entre as abelhas sociais brasileiras e sua contribuição para o entendimento da sua evolução. 
Ciência e Cultura 17: 529-538.

Kerr, W. E. \& H. H. Laidlaw. 1956. General genetics of bees. Advances in Genetics 8: 109-153.

Kerr, W. E.; J. F. Pisani \& D. Aily. 1967. Aplicação de princípios modernos à sistemática do gênero Melipona Illiger, com a divisão em dois subgêneros Hymenoptera, Apoidea). Papéis Avulsos de Zoologia 20: $135-145$.

Lenko, K. \& N. Papavero. 1996. Insetos no folclore. São Paulo, Plêiade, FAPESP, 2a $2^{\text {a }}$ Ed., 468 p.

Lepeletier, A. L. M. 1836. Histoire Naturelle des InsectesHyménoptères. Paris, Roret, Vol. 1, 547 p.

Lutz, F. E. \& T. D. A. Cockerell. 1920. Notes on distribution and bibliography of North American bees of the families Apidae, Meliponidae, Bombidae, Euglossidae, and Anthophoridae. Bulletin of the American Museum of Natural History 42: 491-641.

Marianno, J. 1911. Ensaio sobre as Meliponidas do Brasil. Rio de Janeiro, edição do autor, 140 p.

Melo, G. A. R. 1996. Notes on the nesting biology of Melipona capixaba (Hymenoptera, Apidae). Journal of the Kansas Entomological Society 69: 207-210.

Mercado, N. K. 1968. Contribución al conocimiento de las abejas indígenas (Meloponidae) $[s i c]$ de departamento de Santa Cruz. La Paz, Editorial Don Bosco, 7 p.

Michener, C. D. 1954. Bees of Panamá. Bulletin of the American Museum of Natural History 104: 5-175.

Michener, C. D. 1987. A forgotten paper on South American bees. Melissa 2: 6-7.

Mori, S. A. \& J. J. Pipoly. 1984. Observations on the big bang flowering of Miconia minutiflora (Melastomataceae). Brittonia 36: 337341 .

Moure, J. S. 1944. Abejas del Perú. Boletin del Museo de Historia Natural "Javier Prado" 8: 67-75.

Moure, J. S. 1960. Abelhas da região neotropical descritas por G. Gribodo (Hymenoptera-Apoidea). Boletim da Universidade do Paraná, Zoologia 1: 1-18.

Moure, J. S. 1975. Notas sobre as espécies de Melipona descritas por Lepeletier em 1836 (Hymenoptera - Apidae). Revista Brasileira de Biologia 35: 615-623.

Moure, J. S. 1992. Melikerria e Eomelipona, dois subgêneros novos em Melipona Illiger, 1806 (Hymenoptera, Apidae), p. 32-38. In: C. Cruz Landim \& J. Chaud Netto (eds). Anais do Encontro Brasileiro de Biologia de Abelhas e Outros Insetos Sociais. Homenagem aos 70 anos de Warwick Estevam Kerr. Naturalia, número especial. São Paulo, Editora UNESP, 283 p.

Moure, J. S.; P. Nogueira-Neto \& W. E. Kerr. 1958(1956). Evolutionary problems among Meliponinae (Hymenoptera, Apoidea). Proceedings Tenth International Congress of Entomology 2: 481-493.

Nates-Parra, G. 1983. Abejas de Colombia: I. Lista preliminar de algunas especies de abejas sin aguijón (Hymenoptera: Apidae: Meliponinae). Revista de Biologia Tropical 31: 155-158.

Nates-Parra, G. 1995. Las abejas sin aguijon del genero Melipona (Hymenoptera: Meliponinae) en Colombia. Boletín del Museo de Entomología, Universidad del Valle 3: 21-33.

Nates-Parra, G. 2001. Las abejas sin aguijón (Hymenoptera: Apidae: Meliponini) de Colombia. Biota Colombiana 2: 233-248.

Nates-Parra, G. \& O. I. Cepeda 1983. Comportamiento defensivo en algunas especies de meliponinos colombianos (Hymenoptera: Meliponinae). Boletin del Departamento de Biologia, Universidad Nacional de Colombia 1: 65-82.

Nates-Parra, G. \& V. H. González. 2000. Las abejas silvestres de Colombia: por qué y cómo conservarlas. Acta Biologica Colombiana 5: 5-37.

Nates-Parra, G.; A. Rodríguez-C. \& E. Danny Vélez. 2006. Abejas sin aguijón (Hymenoptera: Apidae: Meliponini) en cementerios de la cordillera oriental de Colombia. Acta Biológica Colombiana 11: 25-35.

Nogueira-Neto, P. 1950. Notas bionomicas sobre Meliponíneos (Hymenoptera, Apoidea). IV. Colônias mistas e questões relacionadas. Revista de Entomologia 8: 305-367.

Nogueira-Neto, P. 1970 a. Behavior problems related to the pillages made by some parasitic stingless bees (Meliponinae, Apidae), p. 416-434. In: L. Aronson, E. Tobach, D. Lehrman \& J. Rosenblatt (eds). Development and evolution of behavior. Essays in Memory of T. C. Schneirla. San Francisco, W. H. Freeman \& Co., 656 p.
Nogueira-Neto, P. 1970 b. A criação de abelhas indígenas sem ferrão (Meliponinae). São Paulo, Tecnapis 2a. Ed., 365 p.

Nogueira-Neto, P. 1997. Vida e criação de abelhas indígenas sem ferrão. São Paulo, Editora Nogueirapis, $446 \mathrm{p}$.

Oliveira, M. L. 2001. Stingless bees (Meliponini) and orchid bees (Euglossini) in terra firme tropical forests and forest fragments, $\mathrm{p}$ 208-218. In: R. O. Bierregaard, Jr., C. Gascon, T. E. Lovejoy \& R. C. G. Mesquita (eds). Lessons from Amazonia. The ecology and conservation of a fragmented forest. New Haven, Yale University Press, Smithsonian Institution, 496 p.

Oliveira, M. L. 2002. As abelhas sem ferrão na vida dos seringueiros e dos Kaxinawá do alto rio Juruá, Acre, Brasil, p. 615-630. In: M. C. Cunha \& M. B. Almeida (eds). Enciclopédia da Floresta. O Alto Juruá: Práticas e conhecimentos das populações. São Paulo, Companhia das Letras, 735 p.

Oliveira, M. L.; E. F. Morato \& M. V. B. Garcia. 1995. Diversidade de espécies e densidade de ninhos de abelhas sociais sem ferrão (Hymenoptera, Apidae, Meliponinae) em floresta de terra firme na Amazônia Central. Revista Brasileira de Zoologia 12: 13-24.

Papavero, N. 1971. Essays on the history of Neotropical Dipterology, with special reference to collectors (1750-1905). Vol. 1. São Paulo, Museu de Zoologia, Universidade de São Paulo, vii +216 p.

Papavero, N. 1999. A "Memoria Acêrca das Abêlhas da Provincia do Piauhí, no Imperio do Brazil" de Leonardo da Senhora das Dores Castello-Branco, (1842), segundo o autógrafo do autor no Instituto Histórico e Geográfico Brasileiro, Rio de Janeiro. I. Introdução e texto. Contribuições Avulsas sobre a História Natural do Brasil, Série História da História Natural 3: 1-10.

Papavero, N. \& D. M. Teixeira. 2000. José Martins Pereira de Alencastre e a fauna do Piauí em 1855, em especial as abelhas. Contribuições Avulsas sobre a História Natural do Brasil, Série História da História Natural 26: 1-5.

Parra, G. 1984. Censo parcial de las abejas sin aguijon (ApidaeMeliponinae) del Occidente Colombiano. Cespedesia 13: 277 290.

Pedro, S. R. M. \& J. M. F. Camargo. 2003. Meliponini neotropicais: o gênero Partamona Schwarz, 1939 (Hymenoptera, Apidae). Revista Brasileira de Entomologia 47: 1-117.

Pereboom, J. J. M. \& J. C. Biesmeijer. 2003. Thermal constraints for stingless bee foragers: the importance of body size and coloration. Oecologia 137: 42-50.

Pierrot, L. M. \& C. Schlindwein. 2003. Variation in daily flight activity and foraging patterns of uruçu - Melipona scutellaris Latreille (Apidae, Meliponini). Revista Brasileira de Zoologia 20: 565 571 .

Pisani, J. F.; W. E. Kerr \& R. A. Cunha. 1977. Estrutura multidimensional dos meliponídeos. Acta Amazonica 7: 233-245.

Pisani, J. F.; W. E. Kerr; B. Crestana; D. Aily \& M. L. Lorenzetti. 1969. Estudo sôbre a estrutura interespecífica de um grupo de espécies do gênero Melipona (Apidae, Hymenoptera). Anais da Academia Brasileira de Ciências 41: 95-107.

Ramalho, M.; V. L. Imperatriz-Fonseca \& A. Kleinert-Giovannini. 1991. Ecologia nutricional de abelhas sociais, p. 225-252. In: A R. Panizzi \& J. R. P. Parra (eds). Ecologia nutricional de insetos e suas implicações no manejo de pragas. São Paulo, Editora Manole Ltda., CNPq, 359 p.

Ramalho, M.; A. Kleinert-Giovannini \& V. L. Imperatriz-Fonseca. 1990. Important bee plants for stingless bees (Melipona and Trigonini) and Africanized honeybees (Apis mellifera) in neotropical habitats: a review. Apidologie 21: 469-488.

Rasmussen, C.; G. Mahé \& I. A. Hinojosa-Díaz. 2007. Taxonomic status of the bees from French Guiana described by Jules Dominique (Hymenoptera: Apidae, Megachilidae, Halictidae). Zootaxa 1423: 59-62.

Rêgo, M. M. C. 1992a. Revisão do gênero Melipona Illiger, 1806: genitalia e esternos pré-genitais de machos (Meliponinae, Apidae, Hymenoptera), [dissertation abstract], p. 382-383. In: Soares, A. E. E. \& De Jong, D. (eds). Pesquisas com abelhas no Brasil. Ribeirão Preto, Revista Brasileira de Genética, 680 p.

Rêgo, M. M. C. 1992b. Morfologia das estruturas genitais de machos na sistemática de Melipona (Meliponinae, Apidae, Hymenoptera), p. 73-78. In: C. Cruz Landim \& J. Chaud Netto (eds). Anais do Encontro Brasileiro de Biologia de Abelhas e Outros Insetos Sociais. Homenagem aos 70 anos de Warwick Estevam Kerr. 
Naturalia, número especial. São Paulo, Editora UNESP, 283 p.

Roubik, D. W. 1979. Africanized honey bees, stingless bees, and the structure of tropical plant-pollinator communities, p. 403-417. In: D. Caron (ed.). Proceedings of the Fourth International Symposium on Pollination. Miscelaneous Publications, Vol. 1. Maryland, Maryland Agricultural Experimental Station.

Roubik, D. W. 1981. A natural mixed colony of Melipona (Hymenoptera: Apidae). Journal of the Kansas Entomological Society 54: 263-268.

Roubik, D. W. 1983. Nest and colony characteristics of stingless bees from Panamá (Hymenoptera: Apidae). Journal of the Kansas Entomological Society 53: 327-355.

Roubik, D. W. 1989. Ecology and natural history of tropical bees. New York, Cambridge University Press, $x+514$.

Roubik, D. W. 1990. Biogeographical ecology of Melipona (Apidae: Meliponinae), p. 579-580. In: G. K.Veeresh, B. Mallik \& C. A Viraktamath (eds). Social insects and the environment: proceedings of the 11 th international congress of IUSSI, Bangalore, India. New Delhi, Oxford \& IBH Publishing Co. Pvt. Ltd., xxxi+765 p.

Roubik, D. W. 1992. Stingless Bees: A guide to Panamanian and Mesoamerican species and their nests (Hymenoptera: Apidae: Meliponinae), p. 495-524. In: D. Quintero, \& A. Aiello (eds). Insects of Panama and Mesoamerica. Selected Studies. Oxford, Oxford University Press, 692 p.

Roubik, D. W. 1993. Direct costs of forest reproduction, bee-cycling and the efficiency of pollination modes. Journal of Biosciences 18: $537-553$.

Roubik, D. W. 1995. Applied pollination in Tropical America, p. 6673. In: D. W. Roubik (ed.). Pollination of cultivated plants in the tropics. FAO Agricultural Services Bulletin, Vol. 118. Rome, Food and Agriculture Organization of the United Nations, $198 \mathrm{p}$.

Roubik, D. W. 1996. African honey bees as exotic pollinators in French Guiana, p. 173-182. In: A. Matheson, S. L. Buchmann, C. O'Toole, P. Westrich \& I. H. Willams (eds). The conservation of bees. San Diego: Linnean Society of London and the International Bee Research Association, 12(s/n) + 254 p.

Roubik, D. W. 2000. Deceptive orchids with Meliponini as pollinators. Plant Systematics and Evolution 222: 271-279.

Roubik, D. W. \& S. L. Buchmann. 1984. Nectar selection by Melipona and Apis mellifera (Hymenoptera: Apidae) and the ecology of the nectar intake by bee colonies in a tropical forest. Oecologia 61: $1-10$.

Roubik, D. W.; J. Enrique Moreno; C. Vergara \& D. Wittmann. 1986. Sporadic food competition with the African honey bee: projected impact on neotropical social bees. Journal of Tropical Ecolology 2: $97-111$.

Roubik, D. W. \& J. E. Moreno. 1990. Social bees and palm trees: what do pollen diets tell us?, p. 427-428. In: G. K.Veeresh, B. Mallik \& C. A.Viraktamath (eds). Social insects and the environment: proceedings of the 11 th international congress of IUSSI, Bangalore, India. New Delhi, Oxford \& IBH Publishing Co. Pvt. Ltd., xxxi+765 p.

Roubik, D. W. \& Q. D. Wheeler. 1982. Flightless beetles and stingless bees: phoresy of Scotocryptine beetles (Leiodidae) on their meliponine hosts (Apidae). Journal of the Kansas Entomological Society 55: 125-135.

Roubik, D. W.; D. Yanega; S. M. Aluja; S. L. Buchmann \& D. W. Inouye. 1995. On optimal nectar foraging by some tropical bees (Hymenoptera: Apidae). Apidologie 26: 197-211.

Sakagami, S. F. 1982. Stingless bees, p. 361-421. In: H. R. Hermann (ed.). Social Insects. New York, Academic Press, Vol. 3.

Sakagami, S. F.; Roubik, D. W. \& Zucchi, R. (1993). Ethology of the robber stingless bee, Lestrimelitta limao (Hymenoptera: Apidae). Sociobiology 21: 237-277.

Schulz, W. A. 1904(1903). Hymenopteren Amazoniens. Sitzungsberichte der mathematisch-physikalischen Klasse der K. B. Akademie der Wissenschaften zu München 33: $757-832$.
Schwarz, H. F. 1932. The genus Melipona the type genus of the Meliponidae or stingless bees. Bulletin of the American Museum of Natural History 63: $231-460$.

Schwarz, H. F. 1934. The social bees (Meliponidae) of Barro Colorado Island, Canal Zone. American Museum Novitates 731: 1-23.

Schwarz, H. F. 1938. The stingless bees (Meliponidae) of British Guiana and some related forms. Bulletin of the American Museum of Natural History 74: 437-508.

Schwarz, H. F. 1944. The stingless bees. A paradox among the producers of honey. Natural History 53: 414-417, 426.

Schwarz, H. F. 1948. Stingless bees (Meliponidae) of the Western Hemisphere. Bulletin of the American Museum of Natural History 90: xvii+546 p.

Serrão, J. E. 2001. A comparative study of the proventricular structure in corbiculate apinae [sic] (Hymenoptera, Apidae). Micron 32: $379-385$

Silveira, F. A.; G. A. R. Melo \& E. A. B. Almeida. 2002. Abelhas Brasileiras: Sistemática e Identificação. Belo Horizonte, F. A. Silveira, 253 p.

Smith, F. 1854. Catalogue of hymenopterous insects in the collection of the British Museum, Part 2. Apidae. London, British Museum, p. 199-465.

Smith-Pardo, A. H. 2003. A preliminary account of the bees of Colombia (Hymenoptera: Apoidea): present knowledge and future directions. Journal of the Kansas Entomological Society 76: 335-341.

Spinola, M. 1840. Observationes sur les apiaires meliponides. Annales des Sciences Naturelles, Zoologie et Biologie Animale 13: $116-140$.

Velthuis, H. H. W. 1976. Environmental, genetic and endocrine influences in stingless bee caste determination, p. 35-53. In: M. Lüscher (ed.). Phase and caste determination in insects. New York, Pergamon Press Oxford \& New York.

Velthuis, H. H. W. 1997. The biology of the stingless bees. Utrecht, Department of Ethology, Utrecht University, The Netherlands and Department of Ecology, University of São Paulo, São Paulo, Brazil, 33 p. +8 plates.

Velthuis, H. H. W.; M. Cortopassi Laurino; Z. Pereboom \& V. L. Imperatriz-Fonseca. 2003. The conservative egg of the genus Melipona and its consequences for speciation, p. 171-176. In: G. A. R. Melo \& I. Alves-dos-Santos (eds). Apoidea Neotropica: Homenagem aos 90 anos de Jesus Santiago Moure. Criciúma, Editora UNESC, $320 \mathrm{p}$.

Vergara, C. B.; A. L. Villa \& G. Nates-Parra. 1986. Nidificación de meliponinos (Hymenoptera: Apidae) de la región central de Colombia. Revista de Biologia Tropical 34: 181-184.

Villa, A. \& C. Vergara. 1982. Contribución al estudio de los hábitos de nidificación de algunas especies de abejas nativas sin aguijón (Hymenóptera, Apidae). Scientia 1: 147-163.

Wille, A. 1961a. Las abejas jicotes de Costa Rica. Revista de la Universidad de Costa Rica 22: 1-30.

Wille, A. 1961b. Evolutionary trends in the ventral nerve cord of stingless bees (Meliponini). Revista de Biologia Tropical 9: 117129.

Wille, A. 1976. Las abejas jicotes del género Melipona (Apidae: Meliponini). Revista de Biologia Tropical 24: 123-147.

Wille, A. 1979. Phylogeny and relationships among the genera and subgenera of the stingless bees (Meliponinae) of the world. Revista de Biologia Tropical 27: 241-277.

Wille, A. 1983. Biology of the stingless bees. Annual Review of Entomology 28: 41-64.

Wille, A. \& C. D. Michener. 1973. The nest architecture of stingless bees with special reference to those of Costa Rica. Revista de Biologia Tropical 21(Suplemento 1): 1-278.

Wille, A. \& E. Orozco. 1975. Observations on the founding of a new colony by Trigona cupira (Hymenoptera: Apidae) in Costa Rica. Revista de Biologia Tropical 22: 253-287.

Zanella, F. C. V.; M. L. Oliveira \& M. C. Gaglianone. 2000. Standardizing lists of locality data for examined specimens in systematics and biogeography studies of New World taxa. Biogeographica 76: $145-160$. 